\title{
Di alcune operazioni atte ad aggiungere o togliere singolarità in una funzione analitica.
}

\author{
(Di S. Pincherle, a Bologna.)
}

Una serie ordinata per le potenze intere positive di una variabile $x$

$$
a_{0}+a_{1} x+a_{2} x^{2}+\cdots+a_{n} x^{n}+\cdots,
$$

definisce, come è noto, una funzione analitica che il metodo detto della continuazione analitica permette di calcolare in tutto il campo della sua validitì. 'Tutte le proprietà della funzione sono dunque contenute nella legge di successione dei coefficienti $a_{n}$ della serie; è però cosa assai difficile, in generale, di mettere in evidenza la dipendenza fra le proprietà della funzione 0 quelle della successione $a_{n}$. Per quanto gli analisti si siano occupati da oltru ad un secolo della questione, pure si æ̌ lungi dal potere rispondere alla scguente domanda:

"Si può, ed in quale modo, da proprietà verificabili sulla successione " dei numeri $a_{n}$, rilevare il numero, la posizione e la natura delle singola"rità della funzione definita dalla serie (1)?"

La prima idea che si presenta alla mente in una simile ricerca, ¿̀ di considerare i coefficienti $a_{n}$ come $\mathrm{i}$ valori di una funzione $a(t)$ di una variabile $t$, per i valori $0,1,2, \ldots$ della variabile stessa. Bene inteso, la funzione $a(t)$ non è definita completamente da queste condizioni, e non lo ̀̀ nemmeno se si astringe ad essere analitica; si possono però aggiungere condizioni opportune, atte a completare convenientemente la determinaziono della $a(t)$. Fra questa, e la funzione $f(x)$ definita dalla serie (1), passa allora una relazione, o corrispondenza funzionale, che è stata considerata gì 
da lungo tempo; essa è stata notata per primo dal LAPLACE $\left(^{*}\right)$, che ha chiamato $f(x)$ funzione generatrice di $a(t)$, e questa, funzione determinante di $f(x)$. Questo autore, e poco dopo di lui, l'Aber (**), hanno sviluppato buon numero di relazioni formali che intereedono fra una generatrice e la sua determinante. Tali ricerche, riprese da varî autori con indirizzo più moderno e coi sussidî piǹ potenti che col Cauchy, col Riemann e col Weierstrass si sono acquistati nella teoria delle funzioni, hanno dato risultati interessanti, fra $\mathrm{i}$ quali mi preme di ricordare $\mathrm{i}$ seguenti :

a) La funzione generatrice si esprime mediante un integrale definitu sotto cui comparisce la determinante, e reciprocamente. In molti casi, l'integrale definito va esteso ad una curva nel piano della variabile d'integrazione, riguardata come complessa. La determinazione di una delle due funzioni, data l'altra, conduce in generale ad un problema d'inversione d'integrale definito.

b) Quando la funzione generatrice soddisfa ad una equazione differenziale lineare a coefficionti razionali in $x$, la determinante soddisfa ad una equazione lineare alle differenze finite, a coefficienti parimente razionali in $t$, e viceversa. Questa osservazione ha permesso di stabilire tipi di equazioni lineari, differenziali o alle differenze $\left(^{* * *}\right)$, integrabili per mezzo di integrali definiti.

Ma risultati non meno notevoli si sono avuti quando la dipendenza ira la successione $a_{n}$ dei coefficienti e la funzione $f(x)$ si è considerata sotto ad un altro punto di vista; quando si è trattato ciò̀ di collegare fra di loro i due seguenti elementi: singolarità della funzione generatrice da una parte, comportamento assintotico della successione dei coefficienti dall'altra. Questo punto di vista interviene per la prima volta in una Memoria del sig. Darboux (****), dove sono ottenuti risultati per alcuni dei casi più semplici; abbandonato per varî anni, esso si presenta di nuovo in lavori recenti dei sigg. HaDA-

(*) Mémoires de l'Académio des Sciences, 1770; Theorie analy tique des probabi-

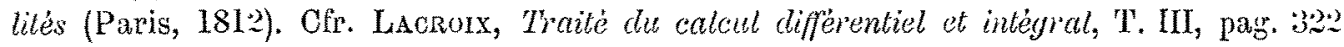
(Paris, 1819).

(*) Euvres, ¿ème éd., T. II, Nóm. XI.

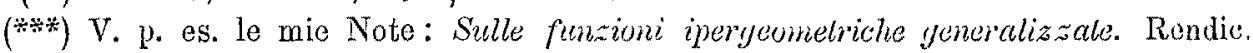
della R. Accad. dei Lincei, 1888, e varie Memorie del sig. Hj. Mebrin negli Acta Math., T. VIII e segg.

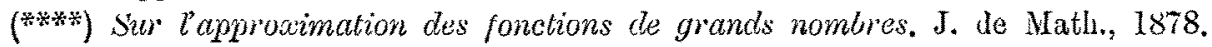


mard $\left({ }^{*}\right)$, Borel $\left(^{* *}\right)$, Fabry $\left({ }^{* * *}\right)$, Le.du $\left({ }^{* * *}\right)$ ed altri. Da questi lavori si può, in casi determinati, dedurre il posto e talvolta la natura delle singolarità della funzione analitica definita dalla serie $\Sigma a_{n} x^{n}$, dal comportamento assintotico della successione $a_{n}$. In tali ricerche non si fa uso ordinariamente della determinante $a(t)$ come funzione analitica di $t$, sebbene i] Bores, nelle osservazioni suggestive della sua Nota citata, abbia consigliato di ricorrervi (*****).

Il presente lavoro si propone di recare una contribuzione alla teoria della lipendenza fra la funzione generatrice e la determinante, studiando alcune operazioni distributive che mentre d'una parte aggiungono, o tolgono, singolarità di specie determinata alla funzione generatrice, rocano d'altra parte alla funzione determinante modificazioni corrispondenti, in guisa che da queste si può dedurre la natura delle singolarità introdotte od eliminate, e reciprocamente. Però, questo studio va preceduto da alcuni paragrafi, nei quali si comyleta in qualche punto la teoria delle operazioni distributive, teoria di cui ho fatto conoscere gli elementi in pubblicazioni anteriori (******). La presente Memoria verrà pertanto divisa in tre capitoli. Nel primo si tratterà della trasformazione di un'operazione mediante un'altra; si considereranno più specialmente quelle operazioni che trasformano una data operazione nell'operazione di moltiplicazione, e si verrà in tal modo ad ottenere (nel modo più elementare ed indipendente da considerazioni d'integrali definiti) l'operazione (che verrà detta operazione $C$ ) che ha la proprietà di mutare ogni generatrice

( $\left.{ }^{*}\right)$ J. de Mathèn., 180:.

(*:3) V. Mémoire ster les séries diveryontes (Ann. de l'Le. Norm., 1880) e Lexons stu la theorie des fonctions (Paris, 1898), passim; a specialmente la Nota dei Cumpl's rendles de l'Acad. des Sciences, liz dóc. 1898.

(***) Ann. de lEEc. Norm., 1896; Acte Math., T. XXII, 1898; Journal de Mathèrin., 1898.

(****) Comptes renclus, 5 décembre.

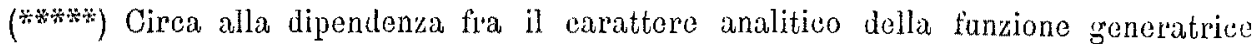
ed il comportamento della sua determinante esistono altri risultati, di natura piu speciale, che non hanno attinenza col presente lavoro, ma che non $\dot{e}$ inutile di ricordare. Quests risultati, ottenuti mediante ricereho ehe si fondano sulla natura aritmetica dolla determinante e che presentano difficolti di altra specie, ma non minori di quelli relativi al comportamento assintotico, si trovano in lavori di Eisensthin, Hane, Tohebitchery, GovesT'eixeira, Pincherle ed altri.

(******) Riassunte in gran parte nel Ménoirc sur le calcul fonctionnel distributif. Matl. Ann., T. XLIX, 1897. 
nella sua determinante. Altre operazioni speciali importanti si presentano eome semplici modificazioni di questa; tale è la trasformazione ben nota di Laplace, - quella di cui il sig. Bores ha recentemente (*) fatto uso nello studio delle trascendenti intere. Il secondo capitolo è dedicato allo studio di operazioni distributive che, per la loro semplicità e simmetria sono sembrate meritevoli del nome di normali; esse si presentano come generalizzazione spontanea di quelle forme differenziali lineari che, uguagliate a zero, dànno le equazioni della classe del Fucns. Di queste operazioni si studiano poi le composizioni fra di loro e con operazioni di moltiplicazione. Infine nel terzo capitolo si tratta dell'argomento principale di questo lavoro: sono definite le singolariti che si dicono semplici per una funzione analitica; si trova che ad ognuna li esse corrisponde un'opernzione $A$, suscettibile di togliere la singolarità; inversamente, l'operazione inversa $A^{-1}$ introduce, nella funzione cui essa si applica, quella singolarita medesima, a meno che la funzione non soddisfi ad una speciale condizione. Ciò accade nel modo stesso che l'operazione di moltiplicazione per $x-z$ toglie un polo di prim'ordine nel punto $x=z$, mentre loperazione di divisione per $x-z$ eseguita su una funzione regolare per $x=z$, vi introduce un polo di prim'ordine in quel punto, a meno che la funzione non soddisfi alla condizione di annullarsi per $x=z$. Le trasformate $C A C^{-1}$ di queste operazioni $A$ mediante l'operazione $C$ definita nella prima parte sono operazioni che portano sulla funzione determinante della furzione $f(x)$ soggetta ad $A$; esse indicano quali modificazioni arrecano ai coefficienti della serie di potenze che rappresenta $f(x)$ la presenza o l'eliminazione della singolarita corrispondente. Infine colla composizione di più operazioni $A$ o delle loro inverse si tolgono o si introducono nelle funzioni analitiche singolarikà più complesse; e si termina coll'applicazione delle cose dette alle singolarità che vengono tolte da forme differenziali lineari della classe del Fucus, o vengono introdotte dalla risoluzione delle equazioni corrispondenti.

(*) Lata Mathematica, T. XXI, 1. 21:3 
atte ad aggiungere o togliere singolarità in una funzione analitica. 223

\section{CAPITOLO I.}

\section{Operazioni trasformatrici.}

\section{Richiamo di al,CUNe proposizioni datia teoria DELLE OPERAZIONI ḊISTRIBUTIVE.}

1. Nelle ricerche che seguono, come in molte altre, è opportuno di considerare le totalità delle serie di potenze di una variabile $x$ come un insieme, o spazio, di cui ogni singola serie costituisce un elemento. Un tale insieme ha evidentemente un numero infinito di dimensioni; esso verrà indieata colla lettera $S$. Ogni serie di potenze si può riguardare come un punto dello spazio $S$, ed i coefficienti della serie si possono riguardare come le coordinate del punto $\left({ }^{*}\right)$.

Ogni serie di potenze ha un cerchio di convergenza di raggio determinato (nullo, finito od infinito). Ci accadrà spesso di dovere considerare l'insieme delle serie di potenze il cui raggio è maggiore del modulo di un numero $x$. Questo insieme verrà denotato con $S_{x}$. È chiaro che se è $|z|>\left|z^{\prime}\right|$, l'insieme $S_{z}$ è contenuto in $S_{z}$. L'insieme delle serie di potenze il cui raggio di convergenza non è nullo, è $S_{0}$.

2. Poichè una funzione analitica, che supporremo ammettere un ramo regolare nell'intorno del punto $x=0$, ̀̀ completamente determinata dal suo sviluppo in serie di potenze nell'intorno di quel punto, cos̀̀ ogni elemento $\alpha(x)$ di $S$, il cui raggio di convergenza non è nullo, definisce una funzione analitica di cui un ramo è regolare nell'intorno di $x=0$. Per schivare le discussioni relative a funzioni non uniformi, sarà opportuno ricorrere al concetto di stella quale è stato recentemente introdotto dal Mitrag-Lefrier. Rimandando, per la definizione di questo concetto, alla sua Memoria (**), con-

(*) V. la mia Nota: Cenno sulla yeometric dello spazio funzionale. Rendic. delli R. Accademia delle Scienze di Bologna, 14 febbraio 1897. Cfr. T. Cazzaxig.1: Intorno ci reciproci dei determinanti normali, pag. 21 . Rendic. della $R$. Accad. delle Scienzo in $T^{\prime}(1-$ rino, 16 aprile 1899 .

(*) MrtTag-LefFlen, sier Ie représentation analytique d'tene branche unifurme d'un" fumlion monogéne. Acta Math., T. XXIII, pag. 4:3, 1899. 
verremo di indicare ad un tempo con $\alpha(x)$ e la serie di potenze di $S$ che si considera, ed il ramo della funzione analitica definita da questa serie esistente ed univocamente determinata nella stella appartenente alla successione $\alpha(0), \alpha^{\prime}(0)\left({ }^{*}\right), \alpha^{\prime \prime}(0), \ldots$

3. E noto che agli elementi di $S$ sono applicabili operazioni che riproducono enti dell'insieme stesso o di un insieme analogo. Fra queste operazioni hanno speciale importanza quelle che ammettono la proprietà distributiva (operazioni distributive) e che vanno riguardate, come ho fatto osservare altre volte, come le omografie dello spazio $S$.

Un'operazione distributiva $A$ è determinata in $S$, quando si conoscono le funzioni che essa fa corrispondere agli elementi $1, x, x^{2}, \ldots x^{n}, \ldots$ di $S$. Porremo:

$$
A\left(x^{n}\right)=\xi_{n}(x), \quad(n=0,1,2, \ldots) .
$$

Si sa allora come ne consegua, per le funzioni $\varphi(x)$ di un insieme convenientemente definito in $S$, lo sviluppo:

$$
A\left(\frac{1}{1}\right)=\sum_{n=0}^{\infty} \frac{x_{n}(x)}{n !} D^{r_{n}}
$$

in cui $D$ ̀̀ l'operazione di derivazione, e le $\alpha_{n}(x)$ sono funzioni dipendenti dalle $\xi_{n}$ mediante le relazioni:

$$
\alpha_{n}=\xi_{n}-n x \xi_{n-1}+\left(\begin{array}{l}
n \\
2
\end{array}\right) x^{2} \xi_{n-2}-\cdots+(-1)^{n} x^{n} \xi_{0} .
$$

Da queste relazioni si ricavano, inversamente, le $\xi_{n}$ in funzione delle $y_{n}$ :

$$
\xi_{n}=\alpha_{n}+n x \alpha_{n-1}+\left(\begin{array}{l}
n \\
2
\end{array}\right) x^{2} \alpha_{n-2}+\cdots+x^{n} \alpha_{0}(* *) \text {. }
$$

4. Le operazioni distributive più semplici sono la moltiplicazione, lit derivazione e la sostiturione.

a) Facendo il prodotto di un elemento arfitrario $\varphi(x)$ di $S$ per una funzione analitica data $\mu(x)$ si ha l'operazione di moltiplicazione, che si rappresenta con $M_{\mu}$. La funzione $\mu(x)$ si può dire moltiplicatore. Consi-

$\left.{ }^{*}\right)$ Con $\alpha^{\prime}(\alpha)$ si indica $\frac{d \alpha}{d x^{*}}$.

(**) V. il mio lavoro: Mémoire su le calcul fonctionnel distributif, Cap. II. Math. Annal., Bd. XLIX. Questo lavoro verri d'ora innanzi citato colla lettera $M$, seguita dal numero del paragrato. 
derando moltiplicatori appartenenti ad $S$, le varie operazioni di moltiplicazione formano un gruppo, sono fra loro commutabili e sono formate colla moltiplicazione $M_{\infty}$ di moltiplicatore $x$; se cioè :

$$
\mu(x)=c_{0}+c_{1} x+c_{2} x^{2}+\cdots,
$$

si avrà :

$$
M_{\mu}=c_{0}+c_{1} M_{x}+c_{2} M_{s}^{2}+\cdots
$$

b) Per la derivazione si userà il solito simbolo $D$. La formola (2) dimostra che ogni operazione distributiva si può esprimere come somma di un numero finito od infinito di prodotti di moltiplicazioni per derivazioni.

c) La sostituzione è l'operazione che consiste nel sostituire alla variabile $x$, in un elemento arbitrario $\subsetneq(x)$ di $S$, la funzione analitica data $\mu(x)$. Questa operazione si rappresenta con $s_{\mu}$. Per essa, lo sviluppo (2) diviene:

$$
S_{\mu}(\varphi)=\sum_{n=0}^{n} \frac{(\mu(x)-x)^{n}}{n !} D^{n}(\varphi) \text {. }
$$

Le operazioni di sostituzione formano un gruppo, non però commutabile.

5. Quando un'operazione è stata definita per gli elementi di uno spazio $S$, ̀̀ spesso possibile di estenderla anche agli elementi di un nuovo spazio $S^{\prime}$ che comprenda $S$; ciò, anche quando la primitiva definizione non sia immediatamente applicabile in $S^{\prime}$. Questa estensione si fa col mezzo del noto principio di permanenza, che l'Hankes $\left(^{*}\right)$ ha enunciato per le operazioni fondamentali dell'Aritmetica, ma che conserva il suo valore in tutte le parti della scienza dei numeri (**). Per applicare questo principio, si procederà in generale nel seguente modo.

Supposto di avere un'operazione $A$ definita per gli elementi di un insieme $S$, sia indicato con a l'insieme di proprietà fra loro indipendenti, di cui gode la $A$ nell'insieme $S$. La definizione data per $A$ in $S$ non sia applicabile ad un nuovo insieme $S^{\prime}$ che comprenda $S$ e sia più esteso. Si possa

(*) Theorie der komplexen Zahlensystemen, \$ 3 (Leipzig, 1867).

(**) E cosi, per esempio, che la derivata si puó definire mediante la regola $D x^{n}=n x^{n-1}$ per tutto l'insieme $S_{0}$. Osservato poi che in questo insieme essa ha la proprietà di essere limite del rapporto incrementale, si viene ad assumere questa proprieti come definizione della derivata in ogni insieme piủ esteso. 
ora, in qualunque modo, definire in $S^{\prime}$ un'operazione $A_{4}$ soggetta alle condizioni :

a) di coincidere con $A$ quando dagli elementi di $S^{\prime}$ si considerano quelli soli che appartengono ad $S$,

b) di godere delle proprietà $a$, che valgono a determinarla in $S$.

Sotto queste condizioni, si riterrà applicabile il sopra citato principio di permanenza e si dirà che la $A_{1}$ è la stessa operazione $A$ estesa al nuovo insieme $S^{\prime}$.

6. L'osservazione precedente permette di estendere a tutto l'insieme delle funzioni analitiche alcune operazioni originariamente definite per il solo spazio $S_{0}$. Questo è manifestamente il caso per le operazioni di moltiplicazione, derivazione e sostituzione; con cio, in $M_{\mu}$ ed $S_{\mu}, \mu$ potrà rappresentare una funzione analitica qualunque, anche non appartenente ad $S_{0}$.

Quando parleremo genericamente di operazioni applicabili all'insieme delle funzioni analitiche, ammetteremo solo che ad una funzione dell'insieme esse facciano corrispondere funzioni dell'insieme stesso. Con spazio funzionale intenderemo poi, sia tutto l'insieme delle funzioni analitiche, sia una parte di esso, colla condizione però che se $\alpha$ e $\beta$ appartengono a questa parte, vi appartenga anche $\alpha+\beta$.

7. Prima di chiudere queste osservazioni preliminari, conviene di notare un altro aspetto che si può dare alle operazioni distributive applicate agli elementi di $S$. Si abbia un'operazione $A$ per la quale sia:

$$
A\left(x^{n}\right)=\xi_{n}(x)=a_{n 0}+a_{n 1} x+a_{n 2} x^{2}+\cdots+a_{n y} x^{\nu}+\cdots
$$

Applicando qucsta operazione all'elemento di $S$ :

$$
\because(x)=k_{0}+k_{1} x+k_{2} x^{2}+\cdots,
$$

ed ammesso che questa serie sia stata presa in modo che risulti uniformemente convergente la $\sum k_{n} \xi_{n}$ in un intorno di $x=0$, si avrà :

$$
A(\rho)=g_{0}+g_{1} x+g_{2} x^{2}+\cdots=\psi(x),
$$

dove i coefficienti $g_{n}$ dipendono dai coefficienti $k_{n}$ per mezzo delle relazioni:

$$
g_{n}=a_{n n} k_{0}+a_{1 n} k_{1}+\cdots a_{v n} k_{n}+\cdots
$$

Sotto questa forma, si vede che all'operazione $A$ che trasforma $\varphi(x)$ in $\psi(x)$, corrisponde un'operazione rappresentata dalla $(6)$, la quale applicata a $l_{n}$, lo trasforma in $g_{n}$; si ha cioè, in altra forma, una trasformazione lineare in uno spazio ad infinite dimensioni. E sotto questa forma che esse sono 
atte ad aggiungere o togliere singolarità in una funzione anulitica. 227

considerate dal sig. Cazzaniga ${ }^{*}$ ) per il caso del determinante normale (d'urdine infinito) non nullo; e a questo proposito notiamo che non sarebbe nè difficile, nè inopportuno, di ricondurre lo studio dei determinanti d'ordine infinito a considerazioni sulle operazioni distributive.

\section{Trasformazioni nelle operazioni.}

8. Siano $A, B$ due operazioni distributive a determinazione unica, definite nell'insieme delle funzioni analitiche. Si dirà che $B$ è trasformuta di $A$ mediante una terza operazione distributiva $X$, quando si abbia:

$$
X A X^{-1}=B \text {. }
$$

Si dirà ancora che $X$ trasforma $A$ in $B$.

In uno spazio funzionale in cui $X$ sia a determinazione unica, la (7) equivale ancora ad:

e a:

$$
\begin{gathered}
X A=B X, \\
X^{-1} B X=A .
\end{gathered}
$$

9. Per vedere quale grado di indeterminazione presenti la $X$ quando siano date $A$ e $B$, si ammetta che $X$ ed $Y$ siano due operazioni che trasformano $A$ in $B$, e si ponga $X=K Y$, onde $X^{-1}=Y^{-1} K^{-1}$ Dalle:

verrà :

$$
X A X^{-1}=B, \quad Y A Y^{-1}=B,
$$

$$
B=K Y A Y^{-1} K^{-4} \text {, onde } B=K B K^{-4} \text {. }
$$

Quest'ultima relazione esprime che $K$ è ćommutabile con $B$; reciprocamente, se $K$ è un'operazione qualunque commutabile con $B$ e $B$ è trasformata di $A$ mediante $X$, lo è anche mediante $K X$.

Analogamente, se in uno spazio in cui $Y$ è a determinazione unica, $B$ è trasformata di $A$ mediante $Y$, lo è anche mediante $Y H$, essendo $H$ un'operazione arbitraria commutabile con $A$; reciprocamente, se $X$ ed $Y$ trasformano $A$ in $B$ e si pone $X=Y H, H$ è commutabile con $A$.

10. Essendo $a_{0}, a_{1}, a_{2}, \ldots$ una successione, finita 0 no, di costanti, è noto che l'operazione:

$$
a_{0} A^{n}+a_{1} A+a_{2} A^{2}+\cdots,
$$

(苂) Nota citata, \& 2 . 
$\grave{e}$, nel suo campo di validità, commutabile con $A$. Ponendo:

$$
f(z)=a_{0}+a_{1} z+a_{2} z^{2}+\cdots,
$$

la (8) si può rappresentare simbolicamente con $f(A)$. Ora:

$$
X A=B X,
$$

onde:

$$
X A^{2}=B X A=B^{2} X
$$

ed in generale, per ogni valore di $n$ intero e positivo:

$$
X A^{n}=B^{n} X
$$

Da ciò (*), sostituendo $A^{0}$ coll'unità :

$$
X\left(a_{0}+a_{1} A+a_{2} A^{2}+\cdots\right)=\left(a_{0}+a_{1} B+a_{2} B^{2}+\cdots\right) X,
$$

0 infine :

$$
X f(A) X^{-1}=f(B) ;
$$

e questa relazione si estende senza difficoltà al caso che $f(A)$ contenga potenze negative di $A$.

La formula (9) di mostra che non solo, mediante una trasformazione $X$, ad operazioni commutabili corrispondono operazioni commutabili (il che $\grave{e}$ affatto ovvio), ma anche che una data funzione di $A\left(^{* *}\right)$ si trasforma nella stessa funzione di $B$.

11. Se $X$ trasforma $A$ in $B$, ed $Y$ trasforma $B$ in $C, Y X$ trasformerà $A$ in $B$. Infatti, da :

$$
X A X^{-1}=B, \quad Y B Y^{-1}=C,
$$

risulta immediatamente :

$$
Y X A X^{-1} Y^{-1}=C \text {. }
$$

Da questo teorema segue che se si ha una classe di operazioni $A, B, C, \ldots$ e si conoscono le operazioni che trasformano un'operazione fissa $P$ nelle operazioni della classe, si conosceranno anche quelle che trasformano le opera-

(*) Nell'applicare l'operazione distributiva ad una serie, conviene tenere presenti le osservationi fatte nel $M ., \S 48$.

(*i) Circa il concetto di funzione di un'operazione, v. Levi-Crvita: Sui gruppi di operazioni funzionali, pag. 4 (Rendie. dell'Ist. Lombardo, T. XXVIII, 1895). 
atte ad aggiungere o togliere singolarità in una funzione analitica. 229

zioni della classe l'una nell'altra. Infatti, si avrà :

$$
\begin{gathered}
X P X^{-1}=A, \quad Y P Y^{-1}=B, \ldots \\
P=X^{-1} A X=Y^{-1} B Y=\cdots,
\end{gathered}
$$

onde, per il teorema precedente, $Y X^{-1}$ trasformerà $A$ in $B$.

12. Sempre nell'ipotesi di limitarci ad un campo funzionale in cui $X$ è univoca, o, come si può anche dire, considerando un ramo univoco della $X$, avremo per il $\$ 10$ :

$$
X A=B X, \quad X A^{2}=B^{2} X, \ldots X A^{n}=B^{n} X, \ldots
$$

Se ora, nello spazio funzionale considerato, $A$ non ha radici ed $\omega$ è una radice di $B$,

$$
A X^{-1}(\omega), \quad A^{2} X^{-1}(\omega), \ldots \quad A^{n} X^{-1}(\omega), \ldots
$$

saranno radici di $X$. Reciprocamente, se $A(\alpha)$ è radice di $X$ senza che to sia $\alpha, X(\alpha)$ sarà radice di $B$ ed $A^{2}(\alpha), A^{3}(\alpha), \ldots A^{n}(\alpha)$, saranno radici di $X$.

\section{LE OPERAZIONI TRASFORMATRICI.}

13. Essendo $M_{\infty}$ l'operazione di moltiplicazione per $x(\S 4)$, chiameremo operazione trasformatrice di un'operazione data $A$ un'operazione $X$ che trasformi $M_{x}$ in $A$. La trasformatrice è dunque definita dall'equazione:

$$
X M_{x} X^{-1}=A \text {. }
$$

In altri termini, se $\varphi$ è un elemento del campo funzionale che si considera, sarà :

$$
X(x \varphi)=A X(\varphi)
$$

e ricordando $\left(^{*}\right)$ che con derivata funzionale di un'operazione $X$ s'intende

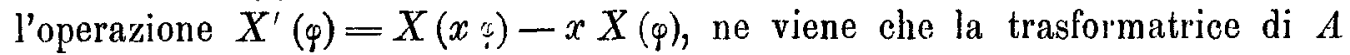
soddisfa all'equazione differenziale simbolica $(* *)$ :

$$
X^{\prime}=(A-x) X
$$

(*) $M ., \S 56$.

(*:) Su queste equazioni differenziali simboliche v.: Di un'eruazione funzionale simbolica, ecc. Rendic. della R. Accad. dei Lincei, 19 febbraio 1809. 
14. Ogni operazione $H$ commutabile con $M_{x}$ ̀̀ una moltiplicazione, poichè la $H M_{s}=M_{x} H$ equivale ad $H^{\prime}=0$, e si è dimostrato che l'operazione, la cui derivata funzionale sia nulla, è una moltiplicazione $\left(^{*}\right)$. Reciprocamente, ogni moltiplicazione è commutabíle con $M_{\mathscr{c}}$. Se dunque si è trovata In trasformatrice $X$ di una data operazione $A$, questa trasformatrice, applicata al gruppo delle moltiplicazioni, lo trasformerà nel gruppo delle operazioni commutabili con $A$.

In particolare, se è :

$$
\mu(x)=a_{0}+a_{1} x+a_{2} x^{2}+\cdots,
$$

si avrà per il $\S 10$ :

$$
X M_{\mu} X^{-1}=\mu(A)
$$

15. La formula precedente, che rappresenta l'enunciato di un teorema per' il caso che il moltiplicatore $\mu$ sia una serie di potenze di $x$, si può assumere como definizione di $\mu(A)$ nel caso che $\mu$ sia una funzione analitica qualunque; potremo dire cioè che con $\mu(A)$ inteuderemo una delle determinazioni di $X M_{\mu} X^{-1}$, essendo $X$ la trasformatrice di $A$.

In particolare,

$$
X\left(x^{m} \varphi\right)=A^{m} X(\varphi)
$$

si potrà prendere come definizione di $A^{n}$ per ogni valore di $m$, scegliendo upportunamente la determinazione di $X(* *)$.

16. Dalle cose dette nel $\S 9$, e dall'osservazione del $\S 14$ che le operazioni commutabili con $M_{\infty}$ sono le moltiplicazioni, risulta che se $X$ è un ramo univoco della trasformatrice di $A$, gli altri suoi rami saranno rappresentati da $X M_{\mu}$, essendo $\mu(x)$ una funzione arbitraria. L'arbitrarietà del fattore $M_{\mu}$ fa si che, avendosi un ramo $X_{1}$ della trasformatrice di $A$ tale che sia $X_{1}(\lambda)=\alpha$, basterà prendere $X_{2}=X_{1} M_{\lambda}$ per avere con $X_{2}$ un ramo della trasformatrice tale che dia $X_{2}(1)=\gamma$. Basterà dunque che a sia nel campo di validita di uno dei rami di $X^{-1}$ per avere un ramo di $X$ che, applicato ad 1 , produce la funzione $a$.

(*) $M ., ॐ 60$.

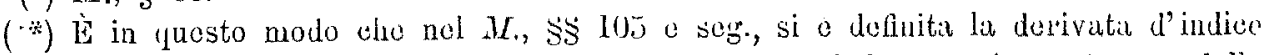
qualunque mediante un'equazione funzionule simbolica che si deduce, in sostanza, dalla tormula (11), V. pii avanti, al $\$ 26$. 
atte ad aggiungere o togliere singolarità in una funzione analitica. 231

17. Passiamo ora alla determinazione effettiva di $X$, supposta data la A. Applicando all' equazione (12) la derivazione funzionale $\left({ }^{*}\right)$, si ottiene senza difficoltà :

quindi :

$$
X^{\prime \prime}=\left(A^{2}-2 x A+x^{2}\right) X,
$$

$$
X^{\prime \prime \prime}=\left(A^{3}-3 x A^{2}+3 x^{2} A-x^{3}\right) X,
$$

e così via. Ponendo:

$$
(A-x)_{m}=A^{m}-m x A^{m-1}+\left(\begin{array}{c}
m \\
2
\end{array}\right) x^{2} A^{m-2}-\cdots+(-1)^{m} x^{m},
$$

il che, bene inteso, non è a confondersi colla $m^{\text {sima }}$ potenza dell'operazione $A-x$, si ha per la derivata funzionale $m^{\text {sima }}$ della $X$ :

$$
X^{(m)}=(A-x)_{m} X
$$

Fatto $X(1)=a$, donde

$$
X^{(m)}(1)=A^{m}(\alpha)-m x A^{m-1}(\alpha)+\cdots+(-1)^{m} x^{m} \alpha,
$$

viene, per la formula $\left(^{* *}\right)$ che dà lo sviluppo di un'operazione distributiva in serie procedente secondo le potenze intere positive della derivata:

$$
X(\xi)=X(1) \varphi+X^{\prime}(1) \varphi^{\prime}+\frac{1}{1.2} X^{\prime \prime}(1) \varphi^{\prime \prime}+\cdots,
$$

ossia :

$$
X(\varphi)=\alpha \varphi+(A-x) \alpha \cdot \varphi^{\prime}+\frac{1}{1.2}(A-x)_{2} \alpha \cdot \varphi^{\prime \prime}+\cdots
$$

Siccome poi per ogni serie di tale forma esiste un campo funzionale di validità, costituito per lo meno dalle funzioni razionali intere, cos̀̀ rimane dimostrata l'esistenza di trasformatrici per ogni operazione $A$. La moltipliciti di determinazioni della $X$ è poi rivelata dall'arbitrarietà della funzione a nella posizione $X(1)=\alpha$; il che è d'accordo con quanto è stato osservato al $\S 16$.

18. Essendo $\alpha$ una funzione qualunque, le $\alpha, \alpha x^{2}, \alpha x^{2}, \ldots$ definiscono uno spazio funzionale che si può chiamare intorno di $\alpha$. Quando un'operazione $K$ ammette come radici lo $a a^{i}$ per $i=0,1,2, \ldots m-1$, ne consegue :

$$
K(\alpha)=K^{\prime}(\alpha)=\cdots=K^{(m-1)}(\alpha)=0,
$$

(*) $M ., \$ 58$.

(**) $M$..,$\$ 63$. 
e lo sviluppo di $K$ in serie ordinata per le potenze di $D$ comincia col termine in $D^{m}$. Quando poi $K$ ammette come radici le $\alpha x^{i}$ per tutti $i$ valori interi positivi di $i$, quello sviluppo cessa di avere significato: in tale caso si potrà dire che $\alpha$ è elemento singolare per $K$, od anche che $K$ è singolare per l'elemento $\alpha$.

19. Ora, le operazioni trasformatrici ammettono, in generale, elementi singolari. A dimostrarlo, consideriamo la trasformatrice $X$ di un'operazione $A$, e sia w una radice di $A$; inoltre, w appartenga al campo di validita di uno lei rami di $X^{-1}$ (il che accadrà in generale) per modo che $X^{-1}(\omega)=\alpha$. Si avrà allora (cfr. § 12):

$$
\begin{gathered}
X(x \alpha)=A(X(\alpha))=0, \quad X\left(x^{\alpha} \alpha\right)=A X(x \alpha)=0, \ldots \\
X\left(x^{m} \alpha\right)=A X\left(x^{m-1} \alpha\right)=0
\end{gathered}
$$

per ogni $m$ intero. L'elemento $x \alpha$ è dunque singolare per $X$. In quanto ad $\alpha$ stessa, esso dà :

$$
X(\alpha)=\omega, \quad X^{\prime}(\alpha)=-x \omega, \ldots \quad X^{(m)}(\alpha)=(-1)^{m} x^{m} \omega,
$$

da cui, essendo $q$ un elemento dell'insieme $S_{0}$ :

$$
X(\alpha \varphi)=\omega\left(\varphi-x \varphi^{\prime}+\frac{x^{2}}{1.2} \varphi^{\prime \prime}-\cdots\right)
$$

cioè, siccome la serie tra parentesi non è altro che la costante $\psi(0)$, l'operazione $X$ fa corrispondere all'intero spazio $\alpha \varphi$, lo spazio ad una dimensione $c \omega$, dove $c$ è una costante arbitraria.

La presenza di elementi singolari nelle operazioni trasformatrici dà, per cosi dire, un carattere di trascendenza a queste operazioni. Infatti le opera. zioni di natura più elementare fra le distributive (come le forme differenziali lineari, le forme lineari alle differenze, le sostituzioni, ecc.) non ammettono elementi singolari.

20. Se $X$ è trasformatrice di $A$, ed $\propto$ non annulla $X$ mentre l'annulla $\propto x, \alpha x$ sarà elemento singolare di $X$. Infatti, dall'equazione di definizione della trasformatrice:

$$
X(x \varphi)=A X(\varphi)
$$

segue, facendo $\Psi=\alpha$, che $X(\alpha)$ è radice di $A$; si ricade quindi nell' ipotesi del paragrafo precedente, ed $X$ è singolare per l'elemento $\alpha x$. 
atte ad aggiungere o togliere singolarità in una funzione analitica. 233

\section{Aldune trasformatrici speciali.}

21. Ci proponiamo ora di dare alcuni esempî di operazioni trasformatrici particolari. Cerchiamo anzitutto la trasformatrice dell'operazione di moltiplicazione $M_{\mu}$. Dovendo essere:

ossia :

$$
X M_{\mathfrak{x}} X^{-1}=M_{\mu}
$$

$$
X(x \varphi)=\mu(x) X(\varphi),
$$

ne verrà, indicando al solito con $X^{\prime}$ la derivata funzionale di $X$ :

$$
X^{\prime}=(\mu(x)-x) X
$$

Ora questa è un'equazione differenziale simbolica appartenente ad una classe di equazioni ch'io ho studiate e risolute in altri lavori $\left({ }^{*}\right)$, dai quali risulta che la soluzione generale dell'equazione (17) è data da $\lambda S_{\mu}$, dove $\lambda$ è una funzione arbitraria ed $S_{\mu}$ è l'operazione di sostituzione di $\mu(x)$ ad $x$ $(\S 4, c)$. Ne viene che la trasformatrice di una moltiplicazione $M_{\mu}$, all'infuori di una moltiplicazione (la cui arbitrarietà risulta dal $\S 16$ ), è la sostituzione corrispondente $S_{\mu}$. Reciprocamente, ogni sostituzione è trasformatrice della moltiplicazione corrispondente.

22. Cerchiamo la trasformatrice della derivazione. Essa sarà definita da:

$$
X M_{x} X^{-1}=D, \quad \text { od } \quad X(x \varphi)=D X(\varphi)
$$

Ne viene che posto $X(\varphi)=\psi$, ed indicate le derivate con accenti, si avrà :

$$
X(\varphi)=\psi, \quad X(x \varphi)=\psi^{\prime}, \quad X\left(x^{2} \varphi\right)=\psi^{\prime \prime}, \ldots
$$

In particolare, possiamo giovarci dell'arbitrarietà che rimane in $X$ secondo il $\S 16$, e porre $X(1)=\alpha$, dove $\alpha$ è una funzione arbitraria; viene allora :

onde :

$$
X(1)=\alpha, \quad X(x)=\alpha^{\prime}, \quad X\left(x^{2}\right)=\alpha^{\prime \prime}, \ldots
$$

$$
\mathrm{X}\left(e^{a x}\right)==\alpha(x+a) \text {. }
$$

23. La formula richiamata al $\S 17$ serve a darci lo sviluppo della trasformatrice di $D$ in serie ordinate per le derivate successive dell'elemento $\varphi$;

(*) Sopra alcune equazioni simboliche. Mem. della R. Accad. delle Scienze di Bologna, S. V, T. V, 189.5. Cfr. anche $M ., \$ 65$ e seg.

Annali di Matematica, Serie III, tomo IV. 
posto $X(\mu)=\alpha$, questa formula diviene:

$$
X(\mu \varphi)=\alpha \varphi+\left(\alpha^{\prime}-x \alpha\right) \varphi^{\prime}+\frac{1}{1.2}\left(\alpha^{\prime \prime}-2 x \alpha^{\prime}+x^{2} \alpha\right) \varphi^{\prime \prime}+\cdots ;
$$

ogni diversa determinazione di $\mu$ ed $\alpha$ ci darà un ramo diverso della trasformatrice di $D$. Esaminiamo alcuni speciali fra questi rami.

24. a) Ponendo dapprima $\mu=1, \alpha=e^{a x}$, viene dalla (22):

$$
X(\varphi)=e^{a x}\left(\varphi+(a-x) \varphi^{\prime}+\frac{(a-x)^{2}}{1.2} \varphi^{\prime \prime}+\cdots\right),
$$

e quindi per lo spazio funzionale $S_{a}$, sarà :

$$
X(\varphi)=e^{a x} \varphi(a) .
$$

Esiste dunque un ramo della trasformatrice di $D$ che fa corrispondere a tutte le funzioni dello spazio $\xi_{a}$ una medesima funzione moltiplicata per una costante, ciò̀ uno spazio ad una sola dimensione $\left(^{*}\right)$. Questo ramo ammette uno spazio di radici ad infinite dimensioni, cioè tutte le funzioni di ${ }_{k}{ }_{a}$ per le quali è $\%(a)=0$.

b) Essendo $\mu$ una funzione arbitraria, poniamo $\alpha=1$. Dalla (19) viene:

$$
X(\mu)=1, \quad X(x \mu)=X\left(x^{2} \mu\right)=\cdots=0 .
$$

La $x \mu$ sarà dunque un elemento singolare per la $X$; ciò proviene dal fatto $(\$ 19)$ che questo ramo della $X$ fa corrispondere a $\mu$ la radice 1 dell'operazione $D$. Applicando nuovamente a questo ramo l'equazione di definizione (18), si ottiene, essendo $a_{0}, a_{1}, a_{2}, \ldots$ costanti arbitrarie introdotte colle successive quadrature:

$$
\begin{gathered}
\mathrm{X}\left(\frac{\mu}{x}\right)=x+a_{0}, \quad X\left(\frac{\mu}{x^{2}}\right)=\frac{x^{2}}{1 \cdot 2}+a_{0} x+a_{1}, \ldots \\
\mathrm{X}\left(\frac{\mu}{x^{n}}\right)=\frac{x^{n}}{n !}+a_{0} \frac{x^{n-1}}{n-1 !}+a_{1} \frac{x^{n-2}}{n-2 !}+\cdots+a_{n-2} x+a_{n-1} .
\end{gathered}
$$

Facendo $\mu=\frac{1}{x}$, e prendendo uguali a zero le costanti d'integrazione, si ottiene una determinazione della $X$ per la quale è :

$$
X\left(\frac{1}{x^{n}}\right)=\frac{x^{n-1}}{n-1 !}
$$

(*) i questa una delle operazioni aventi il massimo tralo di degenerescenza, e che sono indicate colle lettere $C$ noi $\$ \$ 70$ e seguenti del $M$. 
atte ad aggiungere o togliere singolarità in una funzione analitica. 235

c) Poncudo invece $\mu=1, a=\frac{1}{x}$, le (19) dànno:

$$
X\left(x^{n}\right)=(-1)^{n} \frac{n !}{x^{n+1}}
$$

altro ramo della trasformatrice di $D$, pure degno di osservazione.

25. La nota operazione funzionale che va sotto il nome di trasformazione di LAPLace è una delle trasformatrici della derivazione. Infatti, essa si può definire $\left(^{*}\right)$ mediante due equazioni simboliche, una delle quali è precisamente la (18). Essa può anche definirsi come una trasformatrice di $D$, la cui aggiunta (**) è pure trasformatrice di $D\left({ }^{* * *}\right)$. Fra i rami di $X$ enumerati al paragrafo precedente, quelli che soddisfano alle equazioni (23) e (24) sono appunto rami della trasformazione di LAPLACE.

26. Nel $\S 15$ abbiamo definito la funzione $\mu(A)$ di una operazione come risultato della trasformazione $M_{\mu}$ per mezzo della trasformatrice di $A$. Se X è trasformatrice della derivazione, la funzione $\mu(D)$ di $D$ si potrà dunque definire da:

$$
\Upsilon M_{\mu} Y^{-1}=\mu(D) .
$$

In particolare, essendo $r$ un numero qualunque, la $X x^{r} X^{-1}$ si potrà indicare con $D^{r}$. Vi è però in questa posizione l'arbitrarietà dipendente dalla scelta del ramo di $\mathrm{X}$ che figura nella (25). Prendendo in particolare per $X$ la trasformazione di LAPraACE, cioè quella che oltre alla (18) soddisfa anche alla:

$$
X D \varphi=-x X(\varphi)
$$

si trova con un calcolo facile che, posto $X x^{r} X^{-1}=A$, la $A$ soddisferà all'equazione differenziale simbolica $D A^{\prime}=r^{*} A$, che è quella presa al $\S 105$

(*) Auald, Siella trasformazione di Laplace. Rendic, della R. Accad. dei Lincei, s. V, T. VII, 1898.

(*) V. Ia mia Nota: Sulloperazione aggiuntí di una data, nei Rendic. dell'Accad. di Bologna, 17 Aprile 1878.

(*\%) Axaldi (loc. cit.) dimostra che l'aggiunta della trasformazione di LAplack coincide colla trasformazione stessa. Reciprocamente, se si pone che una trastormatrice $X$ della derivazione debba ammettere come aggiunta $\bar{X}$ una trastomatrice della derivaziono, la $\bar{Y}$ dovrà verificare l'equazione $\bar{X}(\mu)=D \bar{X}(p)$, insieme a quella che si ottieno prendendo l'aggriunta dei due membri della (I8), che è (secondo la citata Nota sulloperazione aggiunta) $\bar{Y} D=-i \bar{Y}(p)$ : ora queste due equazioni sono appunto quelle che caratterizzano la trastomazione di LAPLACE. 
del più volte citato Mémoire come equazione di definizione delle derivate d'indice qualunque.

27. Ottenuta la trasformatrice di $D$, ̀̀ facile ottenere lo trasformatrici di operazioni commutabili con $D$, mediante la seguente osservazione generale. Sia $X$ la trasformatrice di un'operazione $A$; si ha:

$$
X M_{\mu} X^{-1}=\mu A \text {. }
$$

Ma al $\S 21$ si è visto che:

$$
S_{\mu} M_{\infty} S_{\mu}^{1}=M_{\mu},
$$

onde:

$$
X S_{\mu} M_{\infty} S_{\mu}^{-1} X^{-1}=\mu(A) .
$$

La trasformatrice di $\mu(A)$ è dunque $X S_{\mu}$. Se dunque $X$ è trasformatrice di $D$, e $\mu(D)$ è un'operazione commutabile con $D$, la regola precedente permette subito di trovarne la trasformatrice.

28. In particolare, assumiamo la trasformazione di Laplace come trasformazione della derivazione ed indichiamola con $L$; essa soddisfa alle due equazioni $(\S 26)$ che la determinano:

$$
L(x \varphi)=D L(\varphi), \quad L D \varphi=-x L(\varphi),
$$

the possiamo anche scrivere:

$$
L M_{x} L^{-1}=D, \quad L^{-1} M_{x} L=-D,
$$

le quali esprimono che $L$ è trasformatrice di $D$ ed $L^{-1}$ di $-D$. Consideriamo ora un'operazione della forma:

$$
K=L S_{\mu} \text {. }
$$

Essa soddisfarà a due equazioni che si deducono dalle (26); la prima sarà :

$$
K M_{x} K^{-1}=\mu(D),
$$

ed esprimerà che $K$ è la trasformatrice dell'operazione $\mu(D)$. In quanto alla seconda, osservo dapprima che la trasformata di $D$ mediante $S_{*}^{-1}$ non è altro che il prodotto dell' operazione $D$ per il moltiplicatore $\nu(x)=\mu^{\prime}\left(\mu_{-1}(x)\right)\left(^{*}\right)$, cioè :

$$
S_{\mu}^{-1} D S_{\mu}=M_{\nu} D
$$

(*) Con $u-1(u)$ indico, secondo una notaziono usuale, la funziono invorsa di $\mu(x)$. 
atte ad aggiungere o togliere singolarità in una funzione analitica. 237

come si verifica subito. Ciò posto, la seconda delle (26) dà :

e quindi :

$$
S_{\mu}^{-1} D S_{\mu}=-S_{u}^{-1} L^{-1} M_{x} L S_{\mu}=-K^{-1} M_{x} K,
$$

$$
K^{-1} M_{x} K=-M, D
$$

Se ne conclude che $K^{-1}$ è la trasformatrice del prodotto della $D$ per il moltiplicatore fisso $\nu$.

29. Di quanto precede si può fare l'applicazione a due casi particolarmente interessanti, il secondo dei quali ci darà quell'operazione dj cui si è fatto cenno nell'introduzione al presente lavoro e che permette (impiegando la terminologia del LAPLACE) il passaggio dalla funzione generatrice alla sua determinante. Di queste operazioni darò qui quel tanto che è necessario per le applicazioni che se ne devono fare, proponendomi di riprenderle in un altro lavoro per uno studio più approfondito.

Il primo caso si ottiene facendo nell'operazione $K=L S_{\mu}^{y}$ del paragrafo precedente, $\mu=\frac{1}{x}$. Indicando con $B$ l'operazione così ottenuta, si trova che per essa le equazioni (27) e (28) divengono:

$$
B M_{x} B^{-1}=D^{-1}, \quad B^{-1} M_{x} B=x^{2} D
$$

l'operazione $B$ è dunque la trasformatrice di $D^{-1}$, e la sua inversa è quella di $x^{2} D$. A questa operazione si potrebbe dare il nome di trasformazione di Bowes, per l'uso sistematico che ne ha fatto questo autore $\left(^{*}\right)$ per trasformare serie di potenze sempre divergenti in serie aventi un raggio di convergenza non nullo, o serie convergenti in un cerchio di raggio finito in serie convergenti in tutto il piano. Un ramo dell'operazione di Bones si ha dal ramo di $L\left({ }^{* *}\right)$ definito dalle equazioni (23) e vale per uno spazio di serie di potenze positive di $x$; esso è definito dalle eguaglianze:

$$
B\left(x^{n}\right)=\frac{x^{n-1}}{n-1 !}
$$

(") Sur les séries de Traylor. Journ. de Math., 1896, Uomptes pondus, 14 décemloro 1896; Sur les séries de Trylor. Acta Math., T. XXI, 189i, otc.

(**) I varì rami delloperaziono di L.ArLuce sono controdistinti dai diver'si spazi funzionali su cui porta l'operazione (v. Axsulu, nota citata). A questi. corrispondono pure varì rami per l'operazione $B$ studiata in cuesto paragrafo e per l'operazione $C$ considerata nel paragrafo successivo, l'una e l'altra dedotte da $L$. Sulla questione del come avvenga il passaggio da un ramo all'altro (passaggio possibile poiché basta uno dei rani a determinare l'operazione) non è qui il luogo di trattenersi. 
un secondo ramo si ha invece dal ramo della $L$ definito dalla (24), e vale per serie di potenze negative di $x$; per esso si ba:

$$
B\left(\frac{1}{x^{n}}\right)=(-1)^{n} \frac{n !}{x^{n+1}} .
$$

Le equazioni di definizione (29) sono evidentemente soddisfatte tanto colle prime formule che colle seconde, mentre esse permettono di definire l'operazione di Bones anche in spazi funzionali in cui le (30) o le (31) non sono applicabili.

30. Il secondo caso particolare cui vogliamo accennare si ottiene $f_{t \prime-}$ cendo nell'operazione $K=L S_{\mu}$ del $\S 28$, la funzione $\mu$ uguale ad $e^{-x}$. Indicando con $C$ l'operazione così definita, le equazioni (26) dànno:

$$
C M_{c} C^{-1}=e^{-D}, \quad C^{-1} M_{x} C=x D .
$$

L'operazione $e^{D}$ essendo quella che muta $x$ in $x+1$ in una funzione di $x$, ciò̀ quella che viene ordinariamente indicata nel calcolo delle differenze col simbolo $\theta$, introdotto dal Casorati, si avrà per la prima delle (32):

$$
C M_{x} C^{-1}=\theta^{-1} \text {. }
$$

Ora, l'operazione che applicata ad una serie di potenze ne dà il coefficiente di $x^{n}$ considerato come funzione dell'indice $\left(^{*}\right),(0$, in altre parole, l'operazione che fa passare dalla funzione generatrice alla sua determinante) gode appunto delle proprietà espresse dalle equazioni $(32)$; e poichè queste equazioni determinano la $C$ come le (26) determinano la $L$, cosil l'operazione in discorso è un ramo dell'operazione $C$ ora definita.

L'operazione $C$ è la trasformatrice di $\theta^{-4}$, mentre la sua inversa ̀̀ la trasformatrice di $x D$. Da ciò la sua proprietà, di trasformare le equazioni differenziali lineari a coefficienti razionali in equazioni lineari alle differenze pure a coefficienti razionali. Tralasciando per ora di insistere sulle proprietà di questa operazione, mi limiterò ad indicare un ramo di essa, valido per l'intorno della funzione esponenziale. Se si pone:

$$
C\left(e^{a x}\right)=a^{x} F^{\prime}(x)
$$

dove $F(x)$ rappresenta il fattoriale, cioè è uguale ad $\frac{1}{\Gamma(x+1)}$, si ha per ogui $n$

(*) Considerata p. us. dal Borks nella nota dei Comptes rendus del 12 dicembre 1898. 
atte ad aggiungere o togliere singolarità in una funzione analitica. 239

intero e positivo, mediante applicazione della prima della (32):

$$
C\left(x^{n} e^{a x}\right)=a^{x-n} F(x) x(x-1)(x-2) \ldots(x-n+1),
$$

e si verifica senz'alcuna difficoltà che in questo modo è soddisfatta anche la seconda delle (32).

\section{CAPITOLO II.}

\section{Operazioni normali.}

\section{Operazioni normali d'ordine nulito.}

31. Definiremo un'operazione distributiva applicabile all'insieme $S$ delle serie di potenze, mediante le equazioni:

$$
U\left(x^{n}\right)=a_{n} x^{n}, \quad(n=0,1,2, \ldots),
$$

dove $a_{0}, a_{1}, \ldots a_{n}, \ldots$ è una successione di numeri dati. Una tale operazione verrà detta normale di ordine nullo; un'operazione normale d'ordine nullo è individuata dalla successione dei numeri $a_{n}$, e le varie operazioni che si possono fare corrispondere alle diverse successioni verranno rappresentate colla lettera $U$, affetta o no da indici. Per brevità, chiameremo operazioni $U$ le operazioni normali di primo ordine.

32. Dalla definizione delle operazioni $U$ risultano immediatamente le seguenti proposizioni :

a) la somma di due o più operazioni $U$ è un'operazione $U$;

b) il prodotto di due operazioni $U$ è un'operazione $U$, e perciò le operazioni $U$ formano un gruppo;

c) il prodotto di due operazioni $U$ è commutativo;

d) una funzione razionale intera a coefficienti costanti di più operazioni $U$ è pure un'operazione $U$; e se indichiamo con $F\left(u_{1}, u_{2}, \ldots u_{r}\right)$ una funzione razionale intera delle indeterminate $u_{1}, u_{2}, \ldots u_{r}$, e poniamo:

si avrà :

$$
U_{i}\left(x^{n}\right)=a_{i n} x_{n}, \quad(i=1,2, \ldots r ; n=0,1,2, \ldots),
$$

$$
F^{\prime}\left(U_{1}, U_{2}, \ldots U_{r}\right)\left(x^{n}\right)=F\left(a_{1 n}, a_{2 n}, \ldots a_{r n}\right) x^{n} .
$$

33. Suppongansi tutti i numeri $a_{n}$ che figurano nelle (1) differenti da zero. L'operazione $U$ non ha allora radici nello spazio $S$; la $U^{-4}$ è a deter- 
minazione unica in questo spazio, ed essendo:

$$
U^{-1}\left(x^{n}\right)=\frac{x^{n}}{a^{2}}
$$

è essa stessa un'operazione $U$. Quando uno dei coefficienti, per esempio $a_{r}$, ¿̀ nullo, l'operazione $U$ ha la radice $x^{r}$; l'operazione $U^{-1}$ ̀े allora a determinazione multipla, poichè si può aggiungere ad una sua determinazione la $r x^{r}$, dove $c$ è una costante arbitraria. Se sono nulli i $p$ coefficienti :

$$
a_{r_{1}}, a_{r_{2}}, \ldots a_{r_{p}},
$$

l'operazione $O$ ammetterà lo spazio di radici ad $r$ dimensioni :

$$
c_{1} x^{r_{1}}+c_{2} x^{r_{1}}+\cdots+c_{p} x^{r_{p}}
$$

colle $p$ costanti arbitrarie $c_{1}, c_{2}, \ldots c_{p}$; e ad una determinazione di $U^{-1}$ si potrà aggiungere la funzione arbitraria (3).

34. Abbiamo visto al $\S 32$ che le operazioni $U$ formano un gruppo commutabile. Fra queste operazioni vi è la $x D$, definita da:

$$
U(1)=0, U(x)=x, \ldots U\left(x^{n}\right)=n x^{n}, \ldots
$$

L'operazione $x D$ ammette come sola radice in $S$ la costante. Ogni operazione $U$ è commutabile con $x D$; recjprocamente, se obblighiamo un'operazione $A$, definita nello spazio $S$ dalle equazioni:

$$
A\left(x^{n}\right)=a_{n 1}+a_{n 1} x+a_{n 2} x^{2}+\cdots+a_{n \nu} x^{\nu}+\cdots,
$$

alla condizione di essere commutabile con $x D$, si vede facilmente che questa condizione porta alla conseguenza che tutti i coefficienti $a_{n y}$ devono essere nulli ad eccezione di $a_{n n}$, e quindi che la $A$ è un'operazione $U$.

35. Fra le operazioni del gruppo delle $U$ si trova la sostituzione $S_{z x}$, definita da:

$$
S_{z x}\left(x^{n}\right)=z^{n} x^{n} .
$$

Considerando, in queste uguaglianze, le $z$ come un parametro arbitrario, queste sostituzioni formano un sottogruppo $\infty^{1}$ del gruppo delle operazioni $U$; si vede subito, posto $z=e^{t}$, che questo sottogruppo si riduce a quello delle traslazioni. Il sottogruppo $S_{z x}$ contiene l'identità per $z=1$; si verifica poi facilmente che la sua trasformazione infinitesima è l'operazione $x D$.

L'operazione $S_{z x} U$ si indicherà con $U_{z}$, e si ha dalle (1) che:

$$
U_{z}\left(x^{n}\right)=a_{n} z^{n} x^{n} .
$$


atte ad aggiungere o togliere singolarità in una funzione analitica. 241

36. Formando le derivate funzionali successive di $U$, si trova:

$$
\begin{aligned}
& U^{\prime}\left(x^{n}\right)=U\left(x^{n+1}\right)-x U\left(x^{n}\right)=\left(a_{n+1}-a_{n}\right) x^{n+1}, \\
& U^{\prime \prime}\left(x^{n}\right)=U^{\prime}\left(x^{n+1}\right)-x U^{\prime}\left(x^{n}\right)=\left(a_{n+2}-2 a_{n+1}+a_{n}\right) x^{n+2},
\end{aligned}
$$

e così via. Analogamente :

$$
\begin{gathered}
U_{z}^{\prime}\left(x^{n}\right)=\left(a_{n+1} z-a_{n}\right) x^{n+1}, U_{z}^{\prime \prime}\left(x^{n}\right)=\left(a_{n+2} z^{2}-2 a_{n+1} z+a_{n}\right) x^{n+2}, \ldots \\
\ldots U_{z}^{(m)}\left(x^{n}\right)=\left(a_{n+m} z^{m}-m a_{n+m-1} z^{m-1}+\right. \\
\left.+\left(\begin{array}{c}
m \\
2
\end{array}\right) a_{n+m-2} z^{m 2}-\cdots+(-1)^{n} a_{n}\right) x^{n+m} .
\end{gathered}
$$

37. Qui sarà conveniente di usare delle notazioni del calcolo delle differenze finite, e di indicare con $\Delta$ la differenza di una funzione della variabile (o indice) $n$. Così $\Delta^{2}, \Delta^{3}, \ldots$ saranno le differenze seconde, terze, ecc., per modo che:

$$
\Delta a_{n}=a_{n+1}-a_{n}, \quad \Delta^{2} a_{n}=a_{n+2}-2 a_{n+1}+a_{n}, \ldots
$$

Con notazione analoga, si indicherà con $\Delta_{z} a_{n}$ il binomio $a_{n+1} z-a_{n}$, ed in generale si porrà:

$$
\Delta_{z}^{m} a_{n}=a_{n+m} z^{m}-m a_{n+m-1} z^{m-1}+\left(\begin{array}{c}
m \\
2
\end{array}\right) a_{n+m-2} z^{m-2}-\cdots+(-1)^{n} a_{n} .
$$

Potremo pertanto scrivere:

$$
U_{z}^{\prime}\left(x^{n}\right)=x^{n+1} \Delta_{z} a_{n}, \ldots \quad O_{z}^{(m)}\left(x^{n}\right)=x^{n+m} \Delta_{z}^{m} a_{n},
$$

ed in particolare :

$$
U^{\prime}(1)=x \Delta_{z} a_{0}, \ldots \quad U_{z}^{(m)}(1)=x^{m} \Delta_{z}^{m} a_{0} .
$$

Richiamando ora la formola che dà lo sviluppo d'una operazione distributiva in serie ordinata per le potenze di $D(\S 3)$, avremo per la $U_{z}$ lo sviluppo :

$$
U_{z}(p)=\sum_{n=0}^{\infty} \frac{x^{n}}{n !} \Delta_{z}^{u} a_{0}+q_{i}^{(n)},
$$

$\mathrm{e}$ in particolare, per $z=1$ :

$$
U(\varphi)=a_{0} \varphi+x \Delta a_{0} \cdot \varphi^{\prime}+\frac{x^{2}}{1.2} \Delta^{2} a_{0} \cdot \varphi^{\prime \prime}+\cdots
$$


Mutando qui $\varphi(x)$ in $\varphi\left(\frac{x}{z}\right)$, si ha per $U(\varphi)$ l'altra espressione:

$$
U(\varphi)=\sum_{n=0}^{\infty} \Delta_{z}^{n} a_{n} \frac{x^{n}}{n !} \frac{d^{n} \varphi\left(\frac{x}{z}\right)}{d x^{n}} .
$$

38. L'operazione $U$ è data, nello spazio $S$ delle serie di potenze, dalle equazioni di definizione (1); da queste risulta formalmente lo sviluppo (6). Limitandosi al punto di vista formale, si può dire che tanto le (1) quanto la (6) definiscono la $U$ per ogni serie di potenze. Si tratta ora di esaminare fino a che punto questo risultato formale abbia un significato effettivo: si è perciò condotti a distinguere due casi, secondo che il sistema dei numeri $a_{n}$ introdotti nelle (1) è tale che la serie $\Sigma a_{n} x^{n}$ abbia, oppure no, un raggio (non nullo) di convergenza.

Nel primo caso, ogni serie $\varphi=\Sigma c_{n} x^{n}$ che abbia un raggio non nullo di convergenza appartiene al campo di validità della operazione $U$. Intendiamo con ciò che tanto le (1) quanto la (6), applicate alla serie $\varphi$, dànno risultato arente significato, ed $\mathrm{i}$ risultati ottenuti per queste due vie coincidono. Infatti, per le ipotesi fatte, essendo $m, m^{\prime}, g, g^{\prime}$, numeri positivi finiti, si avrà :

onde :

$$
\left|a_{n}\right|<m g^{n}, \quad\left|c_{n}\right|<m^{\prime} g^{\prime n}
$$

$$
\left|a_{n} c_{n}\right|<m m^{\prime} g^{n} g^{\prime n}
$$

(*) Considerando due operazioni $U, U_{1}$, date nella forma:

$$
U\left(x^{n}\right)=a_{n} x^{n}, \quad U_{1}\left(x^{n}\right)=a_{n}^{\prime} x^{n},
$$

se ne ricavano le serie:

dove :

$$
U(\varphi)=\Sigma b_{n} \frac{x^{n}}{n !} \varphi^{(n)}, \quad U_{1}(\varphi)=\Sigma b_{n}^{\prime} \frac{x^{n}}{n !} \varphi^{(n)},
$$

$$
b_{n}=\Delta^{n} a_{0}, \quad b_{n}^{\prime}=\Delta^{n} a_{0}^{\prime} .
$$

Formando il prodotto $U U_{1}$, si avri d'una parte:

dall'altra :

$$
U U_{1}\left(x^{n}\right)=a_{n} a_{n}^{\prime} x^{n},
$$

$$
U U_{1}(\varphi)=\Sigma b_{n}^{\prime \prime} \frac{x^{n}}{n !} \varphi^{(n)}, \quad \text { con } \quad b^{\prime \prime}{ }_{n}=\Delta^{n}\left(a_{0} a_{0}^{\prime}\right)
$$

sviluppando il calcolo, si otterrà facilmente per questa via l'espressione di $\Delta^{n}\left(a_{0} a_{0}^{\prime}\right)$ in funzione delle $\Delta^{m} a_{0}, \Delta^{m} a_{0}^{\prime}$ con $m \leq n$. 
atte ad aggiungere o togliere singolarità in una funzione analitica. 243

e la serie $\Sigma c_{n} a_{n} x^{n}$, ottenuta applicando la $U$ alla serie $\varphi$, ha un raggio non nullo di convergenza. Inoltre, $\grave{e}$ :

$$
\left|\Delta^{n} a_{0}\right| \leq\left|a_{n}\right|+n\left|a_{n-1}\right|+\left(\begin{array}{l}
n \\
2
\end{array}\right)\left|a_{n-2}\right|+\cdots+\left|a_{0}\right|
$$

cioè :

$$
\left|\Delta^{n} a_{0}\right|<m(1+g)^{n}:
$$

di più, se $r$ è il raggio di convergenza della serie $\varphi$, per tutti i valori di $x$ il cui modulo è minore di $\frac{1}{2} r$, si ha :

$$
\left|\frac{\rho^{\prime n)}(x)}{n !}\right|<\frac{2^{n} m^{\prime}}{r^{n}}
$$

il termine generale della serie (6) è dunque inferiore a :

$$
\frac{2^{n} m m^{\prime}(1+g)^{n}|x|^{n}}{r^{n}}
$$

e qui basta prendere:

$$
|x|<\frac{r}{2(1+g)}
$$

per rendere la serie (6) convergente assolutamente ed uniformemente. 亡̀ lecito allora, per un noto teorema di WEIenstrass, ordinare il secondo membro della (6) per le potenze di $x$, e si ritrova la $\Sigma c_{n} a_{n} x^{n}$. Ogni elemento di $S_{0}$ appartiene dunque al campo di validità di $U$.

39. Anche nel secondo caso, in cui $\Sigma a_{n} x^{n}$ ha raggio nullo di convergenza, si può assegnare uno spazio $S^{\prime \prime}$ contenuto in $S$ e costituente un campo di validità per l'operazione $U$. Prendiamo, a tale uopo, una successione di numeri $k_{0}, k_{1}, \ldots k_{n}, \ldots$ positivi, decrescenti e soddisfacenti alle disuguaglianze :

$$
k_{n}<\frac{m t^{n}}{\left|a_{n}\right|}
$$

essendo $m$ e $t$ numeri positivi arbitrariamente scelti. Dico che lo spazio $S^{\prime}$ è costituito da tutte le serie:

$$
\varphi=\Sigma c_{n} x^{n}
$$

per le quali è $\left|c_{n}\right|<k_{n}$.

Intanto, ̀̀ manifesto che applicando le (1) ad una tale serie, si ottiene una serie di potenze a raggio di convergenza non nullo. Considerando poi 
la serie (6), si nota che il suo termine generale è, per $|x|<r$, inferiore a:

$$
\frac{r^{n}}{n !}\left(\left|a_{n}\right|+n\left|a_{n-1}\right|+\left(\begin{array}{l}
n \\
2
\end{array}\right)\left|a_{n-2}\right|+\cdots+\left|a_{0}\right|\right) n^{(n)}(r),
$$

dove $n(r)$ è la serie a termini positivi $\sum k_{n} r^{n}$. Essendo la derivata $n^{\text {sima }}$ di questa :

$$
\frac{n^{(n)}(r)}{n !}=k_{n}+(n+1) k_{n+1} r+\frac{(n+1)(n+2)}{1.2} k_{n+2} r^{2}+\cdots
$$

si avrà, per essere le $k_{n}$ decrescenti e per $r<1$ :

inoltre risulta dalla $(8)$ :

$$
\frac{n^{(n '}(r)}{n !}<\frac{k_{n}}{\left(1-r^{n+1}\right.}
$$

$$
\left|a_{n}\right|<m \frac{t_{n}}{k_{n}}, \quad \text { onde } \quad\left|a_{n-1}\right|<m \frac{m^{n-1}}{k_{n-1}}<m \frac{t^{n-1}}{k_{n}},
$$

e quindi :

$$
\left|a_{n}\right|+n\left|a_{n-1}\right|+\left(\begin{array}{l}
n \\
2
\end{array}\right)\left|a_{n-2}\right|+\cdots+\left|a_{0}\right|<m \frac{(t+1)^{n}}{k_{n}} .
$$

L'espressione (9) risulta dunque inferiore ad:

$$
\frac{m(t+1)^{n} r^{n}}{(1-r)^{n}}
$$

e quindi, per $r<\frac{1}{t+2}$, al termine generale di una progressione convergente. Sostituendo ora l'elemento $\varphi$ nella (6), questa serie risulterà uniformemente convergente per $|x|<r$, e perciò sarà trasformabile in una serie di potenze che non potrà differire dalla $\Sigma c_{n} a_{n} x^{n}$.

40. Dalle considerazioni dei due paragrafi precedenti risulta stabilito in ogni caso un campo $S^{\prime}$, contenuto entro $S$, per il quale è valida l'ope. razione $U$ definita dalle (1). Ma se si considera una funzione non appartenente ad $S$, ad esempio la $x^{p}$ per $\rho$ non intero positivo, le formule (1) non saranno applicabili, e l'operazione $U$, in quanto è definita da quella formula, non avrà alcun significato per una tale funzione. Però l'espressione (6), che in $S^{\prime}$ coincide colle (1), può conservare significato anche fuori di $S$. Siamo dunque condotti a considerare la (6) come definizione dell'operazione $U$ in un campo più esteso di quello in cui valeva la definizione originaria (1) (v. $\S 5$ ), e a dire campo di validità $S^{\prime \prime}$ della $U$ così estesa, l'insieme delle funzioni analitiche che, sostituite in (6), la rendono uniformemente convergente 
in tutta un'area connessa nel piano della variabile $x$, quest' area potendo 0 no contenere il punto $x=0$. Evidentemente $S^{\prime}$ fa parte di $S^{\prime \prime}$.

41. Per dare subito un' applicazione di questa estensione dell' operazione $U$, supponiamo il sistema dei coefficienti $a_{n}$ tale che la serie $\Sigma \Delta^{n} a_{0} . x^{n}$ sia convergente in un cerchio di raggio $|z|>1$. Fatta allora $\varphi(x)=x^{p}$, la serie (6) diviene :

$$
U\left(x^{\rho}\right)=x^{\rho} \sum_{i=0}^{\infty} \Delta^{n} a_{0} \frac{p(p-1) \ldots(0-n+1)}{1.2 .3 \ldots n},
$$

e per l'ipotesi fatta sulle $a_{n}$, la serie che qui figura nel secondo membro è una funzione intera di $\rho$, tale da assumere $\mathrm{i}$ valori $a_{0}, a_{1}, \ldots a_{n}, \ldots$ per $\rho=0,1,2, \ldots n, \ldots\left(^{*}\right)$. Sotto le medesime ipotesi per le $a_{n}$, si ha:

dove :

$$
U(\log x)=a_{0} \log x+b,
$$

$$
b=\Sigma(-1)^{n} \frac{\Delta^{n} a_{0}}{n}
$$

42. La proprietà delle operazioni $U$ di essere commutabili con $x D$ (\$34) e che si conferma sulla serie (6), permette pure di ottenere il risultato dell'operazione stessa in uno spazio più esteso di $S$. Questa osservazione può servire a determinare la forma, ad esempio, di $U\left(x^{p}\right)$; si ha infatti :

$$
U\left(x D x^{\rho}\right)=x D U\left(x^{\circ}\right)
$$

ossia, posto $U\left(x^{\rho}\right)=\lambda(x, \rho)$, viene:

da cui :

$$
U\left(\rho x^{\rho}\right)=x \frac{\partial}{\partial x} \lambda(x, \rho), \quad \rho \lambda=x \frac{\partial \lambda}{\partial x},
$$

$$
\lambda(\rho, x)=u(\rho) x^{\prime \prime}
$$

dove $a(\rho)$ è una funzione di $\rho$ ehe, per $\mathrm{i}$ valori interi $\rho=n$, prende $\mathrm{i}$ valori $a_{n}$ dati nelle (1).

43. La trasformata di un'operazione $U$ mediante l'operazione $C$ definita al $\S 30$ (operazione il cui effetto è di fornire la funzione generatrice di una data determinante) è un'operazione di moltiplicazione. Infatti, l'opera-

(*) Essa coincide precisamente, come è facile verificare, collo sviluppo che si ottiane formando col mezzo della formola d'interpolazione di NwwToN estesa all'infinito dal signor Benurxson (Aeta, $T$. IX, 1886) la funzione di $\rho$ che per $\rho=0,1, " 2, \ldots$ assume $i$ valori $a_{0}, a_{1}, a_{2}, \ldots$ 
zione $C$ trasforma $x D$ nella moltiplicazione $M_{x}(\S 30)$ ed $U$ è commutabile con $x D$. Inoltre, mediante una trasformazione, un gruppo commutabile si muta in un gruppo commutabile $(\S 10)$ : onde il gruppo delle operazioni $U$, commutabile con $x D$, viene trasformato da $C$ nel gruppo delle operazioni commutabili con $M_{x}$, le quali ( $\$ 14$ ) non sono altro che le moltiplicazioni.

44. Diamo per ultima un' altra espressione delle operazioni $U$ pure fondata sull' osservazione che esse sono commutabili con $x D$. Ricordiamo perciò $\left({ }^{*}\right)$ che se $A$ e $B$ sono due operazioni distributive commutabili ed $A$ ha una sola radice nel campo di validità di $B$, si ha per $B$ uno sviluppo a coefficienti costanti ordinato per le potenze intere e positive di $A$ :

$$
B=k_{0}+k_{1} A+k_{2} A^{2}+\cdots
$$

Ne viene che un'operazione $U$, poichè è commutabile con $x D$ che ha per sola radice la costante, ammetterà uno sviluppo della forma:

$$
U(\varphi)=k_{0} \varphi+k_{1} x D \varphi+k_{2} \overline{x D}_{\varphi}^{2}+\cdots+k_{n} \overline{x D}_{\varphi}^{n}+\cdots,
$$

che non è difficile di ricondurre alla serie (6), ed il cui campo di validità sarà costituito dalle funzioni $\varphi$ che ne rendono uniformemente convergente il secondo membro.

\section{Risoluzione di Un NOTEvole sistema}

DI INEINITE EQUAZIONI LINEARI AD INEINITE INCOQNITE.

45. Lo sviluppo trovato nel paragrafo precedente per le operazioni $U$ permette di risolvere in modo assai semplice un notevole sistema di infinite equazioni lineari ad infinite incognite, e precisamente il sistema :

$$
\begin{gathered}
k_{0}+k_{1} n+k_{2} n^{2}+\cdots+k_{\nu} n^{y}+\cdots=a_{n}, ; \\
(n=0,1,2,3, \ldots),
\end{gathered}
$$

dove $a_{0}, a_{1}, \ldots a_{n}, \ldots$ sono numcri dati, e $k_{0}, k_{1}, \ldots k_{n}, \ldots$ incognite da determinarsi.

Allo scopo, definiamo un'operazione $U$ mediante le formole (1), dove per le $a_{n}$ si prendano i secondi membri del sistema (12). Avremo per questa $U$ uno

(i) Sulle operazioni distributive commutabili con una operazione data. Atti della R. Accad. di 'Torino, 23 giugno 1895. 
atte ad aggiungere o togliere singolavità in una funzione analitica. 247

sviluppo della forma (11), in cui, facendo $\varphi=x^{n}$, e poichè $\overline{x D}^{m} \cdot x^{n}=n^{m} x^{n}$, viene immediatamente:

onde :

$$
U\left(x^{n}\right)=\left(k_{0}+k_{1} n+k_{2} n^{2}+\cdots+k_{2} n^{2}+\cdots\right) x^{n}
$$

$$
k_{0}+k_{1} n+k_{2} n^{2}+\cdots=a_{n} \text {. }
$$

II sistema (12) sarà dunque risoluto quando si sia trovato lo sviluppo sotto la forma (11) dell'operazione $U$ definita da $U\left(x^{n}\right)=a_{n} x^{n}$.

46. A determinare questo sviluppo, osserviamo che si ha:

$$
\begin{gathered}
x D \log ^{m} x=m \log ^{m-1} x, \ldots \bar{D}^{m} \log ^{m} x=m !, \\
\overline{x D^{m+1}} \log ^{m} x=0,
\end{gathered}
$$

dove con $\log ^{m} x$ s'intende la $m^{\text {sima }}$ potenza del logaritmo. Sostituendo, pertanto, nello sviluppo (11), a $p$ la funzione $\log ^{m} x$, si ottiene:

$$
\begin{aligned}
& U\left(\log ^{m} x\right)=k_{0} \log ^{m} x+m k_{1} \log ^{m-1} x+ \\
& +m(m-1) k_{2} \log ^{m-2} x+\cdots+m ! k_{m} .
\end{aligned}
$$

Ma dallo sviluppo di $U$ sotto alla forma (6), cioè :

$$
U(\varphi)=b_{0}+b_{1} x_{\varphi}+b_{2} \frac{x^{2}}{1.2} \varphi^{\prime \prime}+\cdots,
$$

in cui :

$$
b_{0}=a_{0}, \quad b_{n}=\Delta^{n} a_{0}=a_{n}-n a_{n-1}+\left(\begin{array}{l}
n \\
2
\end{array}\right) a_{n-2}-\cdots+(-1)^{n} a_{n},
$$

dal quale segue immediatamente:

$$
a_{n}=b_{0}+n b_{1}+\left(\begin{array}{l}
n \\
2
\end{array}\right) b_{2}+\cdots+b_{n},
$$

viene, sostituendo $\log ^{m} x$ al posto di $\varphi$ :

$$
U\left(\log ^{m} x\right)=b_{0} \log ^{m} x+\sum_{n=1}^{\infty} \frac{b_{n} x^{n}}{n !} \frac{d^{n} \log ^{m} x}{d x^{n}} .
$$

Si formino ora le derivate successive di $\log ^{m} x$, le quali hanno la forma :

$$
\frac{d^{n} \log ^{m} x}{d x^{n}}=\frac{1}{x^{n}}\left(m p_{n 1} \log ^{m-1} x+m(m-1) p_{n 2} \log ^{m-2} x+\cdots+m ! p_{n m}\right),
$$

dove $p_{n 1}, p_{n 2}, \ldots p_{n m}$ sono coefficienti numerici fra cui sono nulli quelli pei quali è $n<m$. Sostituendo in (14) ed ordinando secondo le potenze del lo- 
garitmo, viene :

$$
\begin{gathered}
U\left(\log ^{m} x\right)=b_{0} \log ^{m} x+ \\
+\sum_{h=1}^{m-1} m(m-1) \ldots(m-h+1)\left(p_{h, h} \frac{b_{h}}{h !}+p_{h, h+1} \frac{b_{h+1}}{h+1 !}+\ldots\right) \log ^{m-h} x .
\end{gathered}
$$

Il confronto di questo sviluppo con (13) dà :

$$
\begin{aligned}
& k_{0}=b_{n}, \\
& k_{1}=p_{11} b_{1}+p_{21} \frac{b_{2}}{2 !}+p_{31} \frac{b_{3}}{3 !}+\cdots \\
& \cdot \cdot \cdot \cdot \cdot \cdot \cdot \cdot \cdot \cdot \cdot \\
& k_{m}=p_{m m} \frac{b_{m}}{m !}+p_{m+1} \frac{b_{m+1}}{m+1 !}+p_{m+2 m} \frac{b_{m+2}}{m+2 !}+\cdots,
\end{aligned}
$$

e queste formule risolvono il sistema. La validità degli sviluppi (15) è subordinata alla condizione che $\log ^{m} x$ si trovi nel campo di validità dello sviluppo di $U$ sotto alla forma (16).

47. Le formule (15) ci dànno le $k_{0}, k_{1}, k_{2}, \ldots$ in funzione delle $b_{0}$, $b_{1}, b_{2}, \ldots$, di cui è nota l'espressione in funzione delle $a_{0}, a_{1}, a_{2}, \ldots$, e viceversa. Si possono quindi prendere come arbitrarie le $b_{n}$ al posto delle $a_{n}$. Si faccia in particolare $b_{n}=z^{n}$, onde $a_{n}=(1+z)^{n}$, e lo sviluppo (6) diverrà :

$$
U(\varphi)=\varphi+z x \varphi^{\prime}+\frac{z^{2} x^{2}}{1.2} \varphi^{\prime \prime}+\cdots
$$

ossia, supposto $x$ non singolare per $\varphi$ e $z$ abbastanza piccolo,

$$
U(\varphi)=\varphi(x+x z) \text {. }
$$

Preso $x$ diverso da zero, $\log ^{m} x$ si trova dunque nel campo di validità di questa $U$, e la (14) non è altro che lo sviluppo di $\log ^{m b}(x+x z)$ in serie di potenze di z. Ma si ha:

$$
\log ^{m}(x+x z)=\log ^{m} x+\sum_{h=1}^{m-1}\left(\begin{array}{c}
m \\
h
\end{array}\right) \log ^{\pi}(1+z) \log ^{m-h} x .
$$

Paragonando colla (13), viene :

$$
k_{h}=\frac{1}{h !} \log ^{h}(1+z),
$$

e poichè i coefficienti numerici $p_{h h}, p_{h+h}, \ldots$ sono indipendenti dalla scelta delle $b_{n}$, risulta che essi non sono altro che i coefficienti dello sviluppo di $\frac{1}{h !} \log ^{h}(1+z)$ in serie di potenze di $z$. Da quanto precede si conclude an- 
atte ad aggiungere o togliere singolarità in una funzione analitica. 249

cora che le serie dei secondi membri delle (15) sono convergenti e risolvono il problema se le $b_{n}$ sono tali che $\Sigma b_{n} t^{n}$ converga in un cerchio di raggio maggior d'uno. La soluzione del problema proposto al $\S 45$ si ha dunque nei seguenti termini:

“Abbiasi il sistema di equazioni lineari nelle infinite incognite $k_{0}$, $k_{i_{1}}, k_{2}, \ldots$

$$
k_{0}+k_{1} n+k_{2} n^{2}+\ldots=a_{n}, \quad(n=0,1,2, \ldots),
$$

e le $a_{n}$ siano tali che, fatto $b_{n}=\Delta^{n} a_{0}$, la serie $\Sigma b_{n} t^{n}$ risulti convergente in un cerchio di raggio maggiore dell'unità. I valori delle incognite sono dati da:

$$
k_{m}=p_{m m} \frac{b_{m}}{m !}+p_{m+1, m} \frac{b_{m+1}}{m+1 !}+\cdots,
$$

dove $p_{m, \nu}$ è il coefficiente di $z^{y}$ nello sviluppo di $\frac{1}{m !} \log ^{m}(1+z)$ in serie di potenze di $z . "$

48. La risoluzione del sistema (12) mediante le (15) dà anche la soluzione di un'altra questione. Riprendiamo i due sviluppi (6) e (11) dell'operazione $U$. Ammettendo ancora che $\Sigma b_{n} t^{n}$ abbia un raggio di convergenza maggiore dell'unità, si avrà dalla $(6)$ :

e la serie:

$$
U\left(x^{\rho}\right)=\left(b_{0}+b_{1} \rho+b_{2}\left(\begin{array}{l}
p \\
2
\end{array}\right)+\cdots\right) x^{f}
$$

$$
a(\rho)=b_{0}+b_{1} \rho+b_{2}\left(\begin{array}{l}
\rho \\
2
\end{array}\right)+\cdots,
$$

è convergente assolutamente ed uniformemente in tutto il piano $\rho$, a quindi sviluppabile in serie di potenze di $\rho$. Ma questa serie si ha immediatamente facendo $\varphi=x^{\circ}$ nella (11), che diviene:

$$
U\left(x^{\rho}\right)=\left(k_{0}+k_{1} \rho+k_{2} \rho^{2}+\cdots\right) x^{p} .
$$

Si trova dunque che fra le $b_{n}$ ed i coefficienti dello sviluppo di $a(\rho)$ in serie di potenze di $\rho$ passano le relazioni (15).

Talchè " fra i coefficienti $k_{n}$, dello sviluppo di una funzione trascendente intera in serie di potenze di $p$ ed i coefficienti $b_{n}$ del suo sviluppo in serie di fattoriali $\left(\begin{array}{l}i \\ n\end{array}\right)$ passano le relazionj $(15)$, equivalenti alle:

$$
k_{0}+k_{1} n+k_{2} n^{2}+\cdots=b_{n}+n b_{n-1}+\left(\begin{array}{l}
n \\
2
\end{array}\right) b_{n-2}+\cdots+b_{0} . "
$$

Annali di Matematica, Serie III, tomo IV. 
III. Operazioni normali d'ordine superiore.

49. Diremo operazione normale d'ordine $m$ l'operazione distributiva $A$ definita, nello spazio $S$ delle serie di potenze, mediante le equazioni:

$$
\begin{gathered}
A\left(x^{n}\right)=\left(a_{n 0}+a_{n 1} x+a_{n 2} x^{2}+\cdots+a_{n m} x^{m}\right) x^{n}, \\
(n=0,1,2, \ldots) .
\end{gathered}
$$

Questa operazione si esprime mediante una serie ordinata secondo le potenze del simbolo di derivazione, nel modo ricordato al $\S 3$. Indicando con $\Delta^{m} a_{i r}$ la differenza finita $m^{\text {sima }}$ :

$$
\Delta^{m} a_{0 r}=a_{m r}-m a_{m-1, r}+\left(\begin{array}{c}
m \\
2
\end{array}\right) a_{m-2, r}-\cdots+(-1)^{m} a_{0 . r},
$$

lo sviluppo in serie di $A$ si ottiene immediatamente sotto la forma:

$$
A(\vartheta)=\sum_{n=0}^{\infty} \frac{x^{n}}{n !} \Delta^{n}\left(a_{n 0}+a_{01} x+\cdots a_{0 m} x^{m}\right) \varphi^{(n)} .
$$

50. L'applicazione termine a termine dell'operazione $A$ ad una serie di potenze $\varphi(x)=\Sigma k_{n} x^{n}$, astraendo per ora dalla questione della convergenza, dà, ordinando il risultato per le potenze di $x$ :

$$
A(\varphi)=\Sigma g_{n} x^{n}=\psi(x)
$$

dove $\mathrm{i}$ coefficienti $g_{n}$ sono legati a $k_{n}$ dalle relazioni:

$$
\left.\begin{array}{l}
g_{0}=a_{00} k_{0} \\
g_{1}=a_{10} k_{1}+a_{01} k_{0} \\
\cdot \cdot \cdot \cdot \cdot \cdot \cdot \cdot \cdot \\
g_{n}=a_{n 0} k_{n}+a_{n-1,1} k_{n-1}+a_{n-2,2} k_{n-2}+\cdots+a_{n-m m} k_{n-m} .
\end{array}\right\}
$$

Ora, la dipendenza espressa da queste formole fra i due sistemi di coefficienti $k_{n}$ e $g_{n}$, è un'operazione distributiva che indicheremo con $\Phi$, e precisamente una forma lineare alle differenze d'ordine $m$ applicata alla funzione $k_{n}$ dell'indice $n$. Questa operazione $\Phi$ non è altro se non la trasformata di $A$ mediante l'operazione $C$ definita al $\S 30$, cioè $\Phi=C A C^{-1}$. Usando il simbolo $\theta$ di Casorati, la forma $\Phi$ si scrive :

$$
\Phi=a_{n 0}+a_{n-1,1} \theta^{-1}+a_{n-2,2} \theta^{-2}+\cdots+a_{n-m m} \theta m \text {. }
$$


atte ad aggiungere o togliere singolarità in una funzione analitica. 251

51. La somma di due operazioni normali del medesimo ordine è una operazione normale dello stesso ordine. La somma di due operazioni normali d'ordine diverso ha l'ordine di quella di ordine maggiore.

52. Il prodotto di due operazioni normali degli ordini $m$ ed $m^{\prime}$ è uria operazione normale dell' ordine $m+m^{\prime}$. L'insieme delle operazioni normali forma dunque un gruppo; non forma invece un gruppo, eccettuato per il caso di $m=0$, l'insieme delle operazioni normali di un dato ordine.

53. Cerchiamo se un'operazione normale può ammettere radici nello spazio $S$. Si dovranno perciò determinare valori di $k_{0}, k_{1}, \ldots k_{n}, \ldots$ tali che sia:

$$
\left.\begin{array}{l}
a_{00} k_{0}=0 \\
a_{10} k_{1}+a_{01} k_{0}=0, \\
\cdot \cdot \cdot \cdot \cdot \cdot \cdot \cdot \cdot \\
a_{n 0} k_{n}+a_{n-1,0} k_{n-1}+\cdots a_{n-m . m} k_{n m}=0
\end{array}\right\}
$$

il che richiede che sia nullo almeno uno dei coefficienti $a_{n j}$. Supponendo tutti i coefficienti $a_{n 0}$ differenti da zero, come faremo d'ora in avanti, l'operazione $A$ è una operazione non degenere nello spazio $S$, e quindi in questo spazio la sua inversa $A^{-1}$ è a determinazione unica.

L'equazione generale del sistema (19) è la $\Phi=0$. Si può soddisfare a questa equazione, dal valore $n=m$ in avanti, dando valori arbitrari a $k_{0}$, $k_{1}, \ldots k_{m-1}$, e si ottiene così una serie di potenze $\omega$ per la quale $A(\omega)$ si riduce ad un polinomio razionale intero arbitrario di grado $m-1$ in $x$.

54. Consideriamo in particolare un'operazione normale di prim'ordine, definita da :

$$
A\left(x^{n}\right)=\left(a_{n}-b_{n} x\right) x^{n}
$$

Per essa, le formule (18) divengono:

$$
\left.\begin{array}{l}
g_{0}=a_{0} k_{0}, \\
g_{1}=a_{1} k_{1}-b_{0} k_{0}, \\
\cdot \cdot \cdot \cdot \cdot \cdot \cdot \cdot \cdot \\
g_{n}=a_{n} k_{n}-b_{n-1} k_{n-1},
\end{array}\right\}
$$

ed il sistema dei coefficienti $k_{n}$ pei quali $A(\psi)$ si riduce ad una costante (che si può senz'altro prendere uguale all'unità) è, ferma sempre l'ipotesi ( $\$ 53$ ) 
delle $a_{n}$ diverse da zero:

$$
k_{n}=\frac{b_{0} b_{1} \ldots b_{n-1}}{a_{0} a_{1} \ldots a_{n-1} a_{n}}
$$

in altri termini, la serie di potenze (della cui convergenza ci si oceuperà pì̀ avanti):

$$
\omega(x)=\frac{1}{a_{0}}+\frac{b_{0}}{a_{0} a_{1}} x+\frac{b_{0} b_{1}}{a_{0} a_{1} a_{2}} x^{2}+\cdots,
$$

è la soluzione, nollo spazio $S$, dell'equazione $A(\varphi)=1$.

55. È possibile di dare una scomposizione delle operazioni normali sotto forma di un prodotto, i cui fattori sono in parte operazioni normali d'ordinc nullo, in parte moltiplicazioni per binomî della forma $z-x$. Cominciando dalle operazioni del prim'ordine, dico che se $A$ è una tale operazione definita dalle (20), si può scomporla nel prodotto:

$$
A=U_{1} M_{z-x} U
$$

dove $U, U$, sono due operazioni normali d'ordine nullo, cd $M_{z \sim x}$, secondo la notazione stabilita al $\S 4$, è la moltiplicazione per il binomio $z-x$, essendo $z$ un numero arbitrario.

All'uopo, scriviamo le equazioni di definizione (1) delle $U, U_{1}$, nella seguente forma:

$$
U\left(x^{n}\right)=p_{n} x^{n}, \quad U_{1}\left(x^{n}\right)=q_{n} x^{n}
$$

ne viene, dalla (24):

$$
A\left(x^{n}\right)=p_{n}\left(z q_{n}-q_{n+1} x\right) x^{n} ;
$$

ora, scrivendo che queste relazioni coincidono colle (20), avremo:

$$
\approx p_{n} q_{n}=a_{n}, \quad p_{n} q_{n+1}=b_{n},
$$

che servono a determinare le successioni $p_{n}, q_{n}$. Si ottiene:

$$
q_{n}=q_{0} z^{n} \frac{b_{0} b_{1} \ldots b_{n-1}}{a_{0} a_{1} \ldots a_{n-1}}
$$

e da questa:

$$
p_{n}=\frac{1}{q_{0}} \cdot \frac{a_{0} a_{1} \ldots a_{n-1} a_{\iota}}{z^{n+1} b_{0} b_{1} \ldots b_{n-1}}
$$

In tal modo è operata la scomposizione dell'operazione di prim'ordine $A$ nella forma (24). I sistemi di numeri $p_{n}, q_{n}$ sono determinati all'infuori della costante arbitraria $q_{0}$. Si noti infine che la serie $\omega(x)$ che soddisfa alla 
atte ad aggiungere o togliere singolarità in una funzione analitica. 253

$A(\varphi)=1$, dipende dai soli coefficienti della $U$, poichè la (23) si può scrivere, facendo $q_{0}=\frac{1}{z}$ :

$$
\omega(x)=\frac{1}{p_{0}}+\frac{x}{p_{1} z}+\frac{x^{2}}{p_{2} z^{z}}+\cdots
$$

56. Una decomposizione analoga alle (24) è possibile per ogni operazione normale d'ordine qualunque $m$. Dimostriamo dapprima che essendo $A$ un'operazione normale d'ordine $m$, si può determinare un'operazione normale $Y$ d'ordine $m-1$ ed un'operazione $U$ d'ordine nullo, in modo tale che sia:

$$
A=Y M_{z-x} U
$$

dove $z$ è un numero dato. Sia infatti $A$ definito dalle $(16) ; Y$ sia definita dalle:

ed $U$ dalle :

$$
Y\left(x^{n}\right)=\left(y_{n 0}+y_{n 1}+\cdots y_{n . m-1} x^{m-1}\right) x^{n},
$$

$$
U\left(x^{n}\right)=\frac{x^{n}}{q^{n}},
$$

dove $y_{n, *}$ e $q_{n}$ sono da determinarsi. Verrà :

$$
\begin{gathered}
Y M_{z-x} U\left(x^{n}\right)=\frac{x^{n}}{q_{n}}\left[z y_{n 0}+\left(x y_{n+1}-y_{n+1 n}\right) x+\right. \\
\left.\left.\quad+\left(z y_{n, 2}-y_{n+1,1}\right) x^{2}+\cdot-y_{n+1, m-1}\right) x^{m}\right]
\end{gathered}
$$

scrivendo ora che queste coincidono colle equazioni (16) che definiseono le $A$, si dovrà porre:

$$
\begin{aligned}
& z y_{n 0}=q_{n} a_{n 0}, \\
& z y_{n, 1}-y_{n+1,0}=q_{n} a_{n 1}, \\
& \cdot \cdot \cdot \cdot \cdot \cdot \cdot \cdot \cdot \cdot \\
& z y_{n, m-1}-y_{n+1, m-2}=q_{n} a_{n m-1}, \\
& \quad-y_{n+1, m-1}=q_{n} u_{n . m} .
\end{aligned}
$$

Dalle $m$ primo fra queste relazioni, si deduce subito:

$$
\left.\begin{array}{l}
y_{n 0}=\frac{q_{n} a_{n 0}}{z}, \\
y_{n, 1}=\frac{q_{n} a_{n 1}}{z}+\frac{q_{n+1} a_{n+1.0}}{z^{2}}, \\
\cdot \cdot \cdot \cdot \cdot \cdot \cdot \cdot \cdot \cdot \cdot \cdot \cdot \\
y_{n, m-1}=\frac{q_{n} a_{n, m-1}}{z}+\frac{q_{n+1} a_{n+1, m-2}}{z^{2}}+\cdots+\frac{q_{n+m-1} a_{n+m-1,0}}{z^{m}}
\end{array}\right\}
$$


e quindi dall'ultima :

$$
a_{n+m n} q_{n+m}+z a_{n+m-1,1} q_{n+m-1}+\cdots+z^{m-1} a_{n+1, m-1} q_{n+1}+z^{m} a_{n m} q_{n}=0 .
$$

Ora questa è un'equazione lineare alle differcnze $\left(^{*}\right)$, dell'ordine $m$, che determina $q_{n}$; ottenute queste, si hanno dalle $\left(28^{\prime}\right)$ le $y_{n o}, y_{n 1}, \ldots y_{n . m-1}$ in funzione delle $a_{n i}$, ed è quindi operata la scumposizione cercata.

In questa scomposizione intervengono $m$ costanti arbitrarie, introdotte dall'integrazione dell'equazione (29): come tali si possono prendere, ad esempio, i valori iniziali $q_{0}, q_{1}, \ldots q_{m-1}$ del sistema delle $q_{n}$.

57. E facile ora di dimostrare che l'operazione normale $A$ di ordine $m$ è decomponibile in un prodotto della forma:

$$
A=U_{m} M_{z_{m}-\infty} U_{m-1} M_{z_{1,},-\infty} \ldots U_{1} M_{z_{1}-\infty} U_{0}
$$

dove $U_{0}, U_{1}, \ldots U_{m}$ sono operazioni normali d'ordine nullo, e $z_{1}, z_{2}, \ldots z_{m}$ sono numeri dati. Infatti, per il caso di $m=1$ il teorema ̀̀ già stato dimostrato al $\$ 55$. Supposta vera per l'ordine $m-1$, la proposizione si estende immediatamente al caso dellordine $m$; infatti, se $a$ è dell'ordine $m$, essa si scompone, per il paragrafo precedente, nella forma:

$$
A=Y M_{z_{1}-\infty} U_{0}
$$

ma per $Y$ vale la scomposizione:

$$
Y=U_{m} M_{\tilde{z}_{i}-x} \ldots U_{1}
$$

onde, sostituendo, si ottiene $A$ sotto la forma del prodotto (30). Le arbitrarie che si introducono in questo modo sono $\frac{m(m+1)}{2}$.

Inoltre, dalle formole (30) si ha immediatamente la decomposizione in prodotto dell'inversa di $A$, ciò̀ :

$$
A^{-1}=U_{0}^{-1} M_{\frac{1}{z_{1}-x}} U_{1}^{-1} \ldots U_{m}^{-1} M_{\frac{1}{z_{m}-x}} U_{m}^{-1} .
$$

58. Presu $U_{0}$ in modo che:

$$
U\left(x^{n}\right)=\frac{x^{n}}{g_{n}}
$$

(*) Si noti clue yuesta equazione $(29)$ coincide colla $\mathbf{D}=0$ del $\$ 50$; basta cambiaro $t_{n}$ in $k_{n} a^{n}$; l'equazione che determina il sistema $q u$ non $\dot{e}$ dunque altro cho yuella che si ottiene uguagliando a zero la trasformata $C A C^{-1}$ di $A$ mediante Poperazione $C^{\prime}$ del $\$ 30$. 
atte ad aggiungere o togliere singolarità in una funzione analitica. 255

dove $q_{n}$ è un integrale della (29), cioè della equazione $\Phi=0$, il metodo del $\S 56$ permette di determinare la $Y$. Come applicazione, se abbiamo ad esempio l'operazione normale di second'ordine definita da:

$$
A\left(x^{n}\right)=\left(a_{n}+b_{n} x+c_{n} x^{2}\right) x^{n},
$$

e poniamo $M_{z_{1}-\infty} U_{0}=A_{1}, U_{z} M_{z_{2}-x} U_{1}=A_{2}$, fissato l'integrale dell'equazione:

$$
\Phi=a_{n+2} q_{n+2}+z_{1} b_{n+1} q_{n+1}+z_{1}^{2} c_{n} q_{n}=0,
$$

che comparisce nelle equazioni di definizione di $U_{0}$, resta determinato $A_{4}$, ed allora le equazioni (28) del $\S 56$ determinano $A_{2}$ senza ambiguità. Essendo $A=A_{2} A_{i}$, si può dire che si è cos̀̀ ottenuto il quoziente (a sinistra) di $A$ per $A_{1}$.

\section{CAPITOLO III.}

\section{Uso delle operazioni normali nel togliere singolarità alle funzioni.}

I. It teorema di Hadamard. Operazionr semplior.

59. Stabiliamo dapprima due notazioni che, per ragione di brevità, useremo in ciò che segue :

a) Con $[a]$ indicheremo il cerchio del piano della variabile $x$, il cui centro è nel punto $x=0$ ed ìl cui raggio è $|a|$.

b) Quando ciò non possa dare luogo ad equivoci, colla stessa scrittura $\alpha(x)$ si rappresenterà tanto una serie di potenze di $x$, quanto il ramo di funzione che nasce dalla continuazione analitica di questa serie in tutta la stella di centro $\left(^{*}\right)$ che le compete.

60. Ricordiamo ora un importante teorema recentemente dato dal signor Hadamard $\left(^{* *}\right)$. Esso si può enunciare come segue:

"Date le serie di potenze:

la serie:

$$
\alpha(x)=\Sigma a_{n} x^{n}, \quad \varphi(x)=\Sigma k_{n} x^{n},
$$

$$
\psi(x)=\Sigma a_{n} k_{n} x^{n}
$$

(*) Secondo la definizione del Mittag-Lefrler. Acta, T. XXIII, p. $4 \pi$.

(**) Comptes rendes, T. CXXIV, p. 192 (1897). Acta Math., T. XXII, p. 5. (1898). 
rappresenta una funzione analitica che ha per punti singolari quelli soli della forma $x=u_{p} v_{q}$, essendo $u_{p}$ i punti singolari della funzione analitica definita da $\alpha(x)$ e $v_{q}$ quelli della funzione definita da $\varphi(x) . "$

La dimostrazione di questo teorema, fondata sulla considerazione di un integrale definito curvilineo, è stata poi ripresa, semplificata ed in qualche punto completata dal sig. Boreu (*) il quale fa anche rilevare che se si riguarda la funzione $\alpha(x)$ come fissa, la $\varphi(x)$ come presa arbitrariamente nell'insieme $S_{v}$, la $\psi(x)$ si può riguardare come risultato di un'operazione distributiva determinata, individuata da $\alpha(x)$ ed applicata a $\varphi(x)$. Poco dopo, io ho notato $\left({ }^{* *}\right)$ che questa operazione è di quelle che nel presente lavoro si sono dette normali d'ordine nullo, ed ho anche indicato come alla dimostrazione del teorema di Hadamard si potesse giungere senza fare uso della considerazione di integrali curvilinei.

61. Data la serie di $S_{0}$ :

$$
\alpha(x)=\sum_{n=0}^{\infty} a_{n} x^{n}
$$

si può, mediante $\mathrm{i}$ suoi coefficienti $a_{n}$, definire un'operazione $U$ dalle:

$$
U\left(x^{n}\right)=a_{n} x^{n} .
$$

Applicando quest'operazione alla serie $\varphi=\Sigma k_{n} x^{n}$, si ottiene come risultato la serie:

$$
U(\varphi)=\psi(x)=\Sigma \alpha_{n} k_{n} x^{n},
$$

pure appartenente ad $S_{0}$ e le cui singolarità in tutto il piano sono nei soli punti indicati dal teorema di Hadamard ora citato (non essendo però escluso che, in casi particolari, qualcuno di questi punti possa anche non essere singolare).

62. Ciò posto, ei occorrerà di considerare operazioni $U^{r}$ per le quali la funzione $\alpha(x)$ che serve a definirle - si noti che è :

$$
\alpha(x)=U\left(\frac{1}{1-x}\right)-
$$

¿̀ particolarmente semplice. Supporremo cioè che la funzione $\alpha(x)$ non abbia a distanza finita che il solo punto singolare $x=1$, per modo che togliendo dal piano il prolungamento del raggio $0 \ldots 1$, da 1 fino all'infinito,

$\left.{ }^{*}\right)$ Bulletin de la Soc. Math. de France, T. XXVI, p. 238, 1898.

(**) Rendiconti alell'Lcoul, rli Bologna, 19 febbraio 1899. 
atte ad aggiungere o togliere singolarità in una funzione analitica. 257

nel piano così tagliato la $\alpha(x)$ rimane priva di singolarità. Quando è tale la funzione $\alpha(x)$, dirò semplice l'operazione $U$ corrispondente.

63. Un'operazione semplice ha le seguenti proprietà:

a) Applicata alla funzione $\varphi(x)$, essa genera, per il teorema di $\mathrm{H}_{\mathrm{A}-}$ DaMard, una funzione che è singolare al più nei punti stessi dove lo è $\varphi(x)$.

b) Il prodotto di due operazioni semplici è un'operazione semplice, cosicchè queste operazioni formano un gruppo.

Dimostreremo, nel paragrafo seguente, come esistano operazioni $U$ che sono semplici, esse e le loro inverse. Per tali operazioni si hanno le proprietà seguenti :

a) Applicandole ad una funzione $\varphi(x)$, si genera una funzione singolare in tutti e soli $\mathrm{i}$ punti in cui è singolare $\varphi(x)$ : tali operazioni, nelle funzioni cui si applicano, non possono dunque nè aggiungere, nè togliere singolarità.

b) Le operazioni semplici insieme alle loro inverse, formano un gruppo.

64. Per mostrare l'esistenza di operazioni le quali sono semplici unitamente alle loro inverse, consideriamo la forma differenziale lineare d'ordine arbitrario $m$ :

$$
F(\varphi)=b_{0} \varphi+b_{1} x \varphi^{\prime}+b_{2} \frac{x^{2}}{1.2} \varphi^{\prime \prime}+\cdots+b_{m} \frac{x^{m}}{m !} \varphi^{(m)} \text {. }
$$

Questa forma ci dà un'operazione $U$ per la quale è:

$$
U\left(x^{n}\right)=a_{n} x^{n}
$$

con :

$$
a_{n}=b_{0}+n b_{1}+\left(\begin{array}{l}
n \\
2
\end{array}\right) b_{2}+\cdots+\left(\begin{array}{c}
n \\
n
\end{array}\right) b_{m} ;
$$

le $a_{n}$ sono cioè polinomî razionali interi di grado $m$ rispetto all'jndice $n$. Reeiprocamente, ogni $U$ le cui $a_{n}$ sono tali polinomi si riduce, come risulta dalla formula (6) del $\S 37$, ad una forma differenziale lineare del tipo (4). Supponiamo ora che le $a_{n}$ sieno diverse da zero per ogni valore intero positivo o nullo dell'indice. Siccome la $F\left(\frac{1}{1-x}\right)$ è una funzione razionale col solo polo $x=1$, la $F$ è intanto un'operazione semplice. Inoltre, poichè le $a_{n}$ sono diverse da zero, risulta dai principî generali della teoria delle equazioni differenziali lineari che l'equazione:

$$
F(\varphi)=\frac{1}{1-x}
$$

ammette una soluzione regolare nell'intorno di $x=0$, e che non ha singoAmali di Matematica, Serie III, tomo IV. 
larità a distanza finita altro che nel punto $x=1$. Ciò equivale a dire che è tale la funzione $U^{-1}\left(\frac{1}{1-x}\right)$, cioè che $U^{-1}$ è un'operazione semplice. La (4) definisce dunque un'operazione semplice, insieme alla propria inversa.

65. Dimostrato nel paragrafo precedente che sono semplici insieme alla propria inversa quelle operazioni $U$ in cui $a_{n}$ è funzione razionale intera dell'indice, ne viene che sono tali anche le operazioni in cui $\frac{1}{a_{n}}$ è funzione razionale intera dell'indice. $\mathrm{E}$ poichè le operazioni che ammettono la proprietà indicata formano un gruppo, concluderemo che:

" Ogni operazione $U$, definita da $U\left(x^{n}\right)=a_{n} x^{n}$, dove $a_{n}$ è una funzione razionale intera o fratta dell'indice $n$, non nulla nè infinita per valori interi positivi dell'indice stesso, è un'operazione semplice ed è tale la sua inversa."

66. Ho chiamato (*) funzione analitica semplice una funzione che a distanza finita è singolare in un solo punto, ad esempio $x=z$ ( $z$ differente da zero) per modo che togliendo dal piano il prolungamento da $z$ all'infinito del raggio $0 z$, la funzione si riduce regolare ed uniforme nel piano così tagliato. Ogni tale funzione dà lungo ad una operazione semplice: se infatti $\Sigma a_{n} x^{n}$ è lo sviluppo della funzione nell'intorno di $x=0$, l'operazione semplice in discorso è definita da:

$$
U\left(x^{n}\right)=a_{n} z^{n} x^{n}
$$

\section{Uso delLe operazioni Normali di Prim'ordine PER TOGLIERE SINGOLARITA.}

67. Un'operazione normale di prim'ordine è definita nello spazio $S$ dalle relazioni, dove le $a_{n}$ sono tutte diverse da zero:

$$
A\left(x^{n}\right)=a_{n} x^{n}-b_{n} x^{n+1}
$$

abbiamo poi dimostrato $(\S 55)$ che ogni tale operazione si può porre sotto la forma:

$$
A=U_{1} M_{z \cdot x} U
$$

dove $U, U_{1}$ sono operazioni normali d'ordine zero, definite da:

$$
U\left(x^{n}\right)=p_{n} x^{n}, \quad U_{1}\left(x^{n}\right)=q_{n} x^{n} .
$$

(*) Rendiconti della R. Licad. clei Lincei, 16 marzo 1800. 
atte ad aggiungere o togliere singolarità in una funzione analitica. $\quad 259$

Nel presente articolo, daremo le proprietà di quelle operazioni $A$ per le quali è possibile una scomposizione nella forma (6) tale che $U$ ed $U_{1}$ siano operazioni semplici $(\S 62)$, esse e le loro inverse: proprietà che conducono a risultati non indegni di nota circa alle singolarità isolate delle funzioni analitiche.

68. L'operazione $\boldsymbol{A}$ applicata ad una funzione analitica regolare nell'intorno $[r]$ di $x=0$, genera una funzione analitica regolare almeno in quello stesso intorno. Ciò consegue immediatamente dalla forma (6) dell'operazione $A$ e dall'essere $U, U_{1}$ operazioni semplici. Ci proponiamo ora la seguente questione :

"Posto $A(\varphi)=\psi$, se $\varphi$ è una serie di potenze il cui cerchio di convergenza è $[r]$, può $\psi$ appartenere ad $S_{r}\left({ }^{*}\right)$, e sotto quali condizioni? n

La risposta a questa questione forma l'oggetto dei seguenti paragrafi.

69. Poichè ogni costante appartiene ad $S_{r}$, cominciamo dal considerare l'equazione funzionale:

$$
A(\rho)=1 \text {, }
$$

dove $\varphi$ è la funzione incognita. Una soluzione della (7) è data al $\S 55$ : essa è rappresentata dalla serie:

$$
\omega(x)=\frac{1}{p_{0}}+\frac{x}{p_{1} z}+\frac{x^{2}}{p_{2} z^{2}}+\cdots=z U^{-1}\left(\frac{1}{z-x}\right),
$$

e poichè le $a_{n}$ si sono supposte tutte diverse da zero, questa soluzione è unica nello spazio $S$. Ora, poichè $U$ soddisfa alla condizione d'essere semplice insieme alla sua inversa, ne viene che la serie:

$$
\Sigma \frac{x^{n}}{p_{n}}
$$

converge entro il cerchio [1] ed ha sulla circonferenza il solo punto singolare $x=1$; onde $\omega(x)$ è regolare entro $[z]$ ed ha sulla circonferenza il solo punto singolare $x=z$. Questa serie $\omega(x)$, che rappresenta una funzione analitica semplice, serve a rispondere affermativamente alla domanda posta in fine del $\S 68$, poichè la serie stessa $\omega$ converge entro [z], mentre $A(\omega)$ appartiene ad $S_{z}$.

(*) Ricordiamo che si $\dot{\theta}$ convenuto, al $\S 1$, di indicare con $S_{z}$ l'insieme delle serie di potenze il cui cerchio di convergenza è superiore a $|z|$. 
70. Si osservi che il fattore simbolico $U_{1}$ della $A$ non ha alcuna importanza per la risoluzione dell' equazione funzionale $(7)$ : risoluta cioè per l'operazione $M_{z-\infty} U$, essa è risoluta per ogni operazione $U_{i} M_{z-x} U$, essendo $U_{i}{ }^{\prime}$ un'operazione qualunque d'ordine nullo.

Si osservi ancora che per avere la soluzione generale dell'equazione (7), basta aggiungere ad $\omega$ una radice qualunque di $A$; queste soluzioni non verranno però considerate per ora, poichè ci limitiamo qui alla considerazione di funzioni appartenenti ad $S$, ed $A$, come si è già notato, non ha radici in $S$.

71. Poniamo ora la seguente definizione. Due serie di potenze $\varphi=\Sigma k_{n} x_{n}$, $\xi_{1}=\sum_{n}^{\prime} x^{n}$, aventi lo stesso cerchio di convergenza $[r]$, si diranno ugualmente singolari sulla circonferenza $\left({ }^{*}\right)$ di quel cerchio, quando esista una costante $c$ tale che la serie $\varphi-c p_{1}$ abbia un cerchio di convergenza maggiore di $[r]$, cioè appartenga ad $S_{r}$.

La condizione affinchè $\varphi, \varphi_{1}$ siano ugualmente singolari è che esistano due numeri positivi $m$ ed $r^{\prime}$, con $r^{\prime}>|r|$ e tali che sia da un valore $n_{1}$ di $n$ in poi :

$$
\left|k_{n}-c k_{n}^{\prime}\right|<\frac{m}{r^{\prime n}} .
$$

E chiaro che ogni operazione $U$ semplice insieme alla sua inversa, trasforma due serie ugualmente singolari in due serie ugualmente singolari.

Si ha immediatamente che due serie ugualmente singolari con una terza sono ugualmente singolari fra loro.

72. Se $\varphi(x)$ è una serie di potenze convergente entro [z] ed ugualmente singolare sulla circonferenza colla soluzione a dell'equazione $(7), A(\varphi)$ appartiene ad $S_{z}$. Infatti, esiste per ipotesi una costante $c$ tale che:

$$
\varphi=k \omega+\rho
$$

dove $\rho$ è una serie di $S_{z}$; ne viene:

$$
A(\varphi)=7+A(\rho)
$$

e siccome $A(\rho)$ appartiene ad $S_{z}$, la proposizione è dimostrata. Questa funzione $\varphi(x)$ risponde dunque alla questione posta al $\S 68$.

Reciprocamente, tutte le funzioni che rispondono a quella questione sono ugualmente singolari con $\omega$, e quindi fra di loro. Sia infatti $q$ una serie di

(i) Quando ció non possa generare equivoco, si ommetteranno le parole: sulla circonferenza. La definizione di serie di potenze ugualmente singolari è stata introdotta in una Nota che lo pubblicata nei Rendic. dell'Accad. di Bologna, 30 gennaio 1898. 
atte ad aggiungere o togliere singolarità in una funzione analitica. 261

potenze avente $[\vartheta]$ per cerchio di convergenza, ed $A(\varphi)=\rho$ appartenga ad $\boldsymbol{S}_{r}$; vi apparterrà anche $U_{1}^{-1}(\rho)=\rho_{1}$, e si avrà :

$$
\varphi=A^{-1}(\rho)=U^{-1} M_{\frac{1}{z-x}} U_{1}^{-1}(\rho)=U^{-1}\left(\frac{\rho_{1}}{z-x}\right)
$$

Per l'ipotesi che $U^{-1}$ è operazione semplice, risulta da questa ultima eguaglianza che $\varphi$ e $\frac{\rho_{1}}{z-x}$ sono singolari, negli stessi punti; ma non converge oltre il cerchio $[r]$, mentre $\rho_{1}$ converge entro un cerchio maggiore, perciò deve necessariamente essere $|r|=|z|$ e $\rho, \rho_{1}$ appartengono ad $S_{z}$. Ciò posto, $\rho_{1}$ convergendo in un cerchio maggiore di $[z]$, viene:

$$
\rho_{1}(x)=\rho_{1}(z)+(z-x) \rho_{2}(x),
$$

dove anche $\rho_{2}$ appartiene ad $S_{z}$. Ne viene:

$$
\varphi=U^{-1}\left(\rho_{2}(x)\right)+\rho_{1}(z) U^{-1}\left(\frac{1}{z-x}\right)
$$

ma $U^{-1}\left(\rho_{z}(x)\right)=\rho_{3}(x)$, dove $\rho_{3}$ appartiene ad $S_{z} ;$ inoltre :

$$
\omega(x)=z U^{-1}\left(\frac{1}{z-x}\right) \text {, }
$$

onde, indicando con $c$ la costante $z p_{1}(z)$, viene:

$$
\varphi=p_{3}+c \omega,
$$

ciò̀ $\varphi$ è singolare come $\omega$, c. d. d.

73. Dai paragrafi precedenti risulta ehe, data un'operazione normale di prim'ordine i cui fattori $U, U$, siano semplici insieme alle loro inverse, resta stabilita una speciale singolarità nel punto $z$, singolarità definita dalla serie :

$$
\omega(x)=U^{-1}\left(\frac{1}{z-x}\right)=\sum_{n=0}^{\infty} \frac{x^{n}}{p_{n} z^{n+1}},
$$

la quale serie definisce una funzione regolare in tutto il piano tagliato sul prolungamento del raggio $0 z$, fra $z$ ed $\infty$. L'operazione $A$ ha la proprietà di fare sparire la singolaritò cos̀̀ definita; cioè, se $\varphi$ è regolare in $[z]$ e singolare sulla circonferenza come la $\omega, A(\varphi)$ è regolare oltre $[z]$; e reciprocamente, ogni funzione la cui singolarità è tolta da $A$, è singolare in $z$ come lo è come la $\omega$. 
Inversamente, data una funzione semplice:

$$
\omega(x)=\sum \frac{x^{n}}{p_{n} z^{n}}
$$

col solo punto singolare $z$ a distanza finita, la funzione $\Sigma \frac{x^{n}}{p_{n}}$ sarà semplice col solo punto singolare $x=1$, e per il teorema di Hadamard sarà tale, in generale $\left({ }^{*}\right)$, anche la $\Sigma p_{n} x^{n}$. In tale caso la $U$ definita da:

$$
U\left(x^{n}\right)=p_{n} x^{n}
$$

sarà un'operazione semplice insieme alla sua inversa, e le singolarità della $\omega(x)$ sarà tolta dall'operazione di primo ordine $A=M_{z-\infty} U$.

74. Ricordando le relazioni date al $\$ 55$ fra le $a_{n}, b_{n}$, coefficienti delle $A\left(x^{n}\right)$, e le $p_{n}, q_{n}$ coefficienti rispettivi in $U\left(x^{n}\right), U_{1}\left(x^{n}\right)$, le quali relazioni sono:

$$
z p_{n} q_{n}=a_{n}, \quad p_{n} q_{n+1}=b_{n}
$$

si trova che la serie $\omega(x)$, che dà il tipo delle singolarità che l'operazione $A$ è suscettibile di togliere, si scrive:

$$
\omega(x)=z q_{0} \sum_{n=0}^{\infty} \frac{b_{0} b_{1} \ldots b_{n-1}}{a_{0} a_{1} \ldots a_{n}} x^{n} .
$$

75. Applicando l'operazione $A$ ad una serie di potenze $\varphi(x)=\Sigma k_{n} x^{n}$, si ottiene una serie di potenze $\psi(x)=\Sigma g_{n} x^{n}$, i cui coefficienti $g_{n}$ sono legati a $k_{n}$ dalle relazioni:

$$
g_{0}=k_{0} a_{0}, \quad g_{n}=a_{n} k_{n}-b_{n-1} k_{n-1} .
$$

La $g_{n}$ è dunque data dall'applicazione di una forma lineare alle differenze dal primo ordine sulla funzione di $n, k_{n}$; indicando questa forma con:

$$
\Pi\left(k_{n}\right)=a_{n} k_{n}-b_{-1} k_{n-1},
$$

essa è ( $\$ 50$ ) la trasformata di $A$ mediante l'operazione $C$ definita al $\S 30$, cioè $\Pi=C A C^{-1}$. Sostituendo alle $a_{n}, b_{n}$, le loro espressioni (9) nelle $p_{n}, q_{n}$, la II si scrive ancora:

$$
\Pi\left(k_{n}\right)=q_{n}\left(p_{n} z k_{n}-p_{n-1} k_{n \cdot 1}\right) .
$$

76. Condizione necessaria e sufficiente affinchè una serie di potenze $\Psi(x)=\Sigma k_{n} x^{n}$ di cui $[z]$ è il cerchio di convergenza, sia singolare come $\omega(x)$

(*) Boras, Nota citata del Bulletin de la Soc, Mathém., introduzione. 
atte ad aggiungere o togliere singolarità in una funzione analitica. 263

sulla circonferenza, è che per $\mathrm{i}$ valori dell'indice $n$ superiori ad un numero dato $n$, sia :

$$
\left|\frac{p_{n}}{p_{n+1} z} k_{n}-k_{n+1}\right|<\frac{m n^{n}}{|z|^{n}}
$$

essendo $m$ ed $n$ due numeri positivi ed $n<1$; oppure, ciò che torna lo stesso, che sia per $n>n_{1}$ :

$$
\left|\sqrt[n]{\frac{p_{n}}{p_{n+1}} z k_{n}-k_{n+1}}\right|<a<\frac{1}{|z|}
$$

la condizione (13) equivale ancora alla disuguaglianza:

$$
\left|\frac{b_{n}^{n}}{a_{n+1}} k_{n}-k_{n+1}\right|<\frac{m n^{n}}{|z|^{n}} \text {. }
$$

La condizione enunciata è necessaria. Infatti, sia $\varphi(x)$ singolare come $\omega(x)$ sulla circonferenza del cerchio $[z]$ : esisterà un numero $c$ tale che :

$$
\varphi(x)-c \omega(x)
$$

sia una serie $\rho(x)$ di $S_{z} ;$ il coefficiente di $x^{n}$ in $\rho(x)$, cioè :

$$
k_{n}-c \frac{b_{0} b_{1} \ldots b_{n-1}}{a_{0} a_{1} \ldots a_{n}}
$$

sarà, in valore assoluto, inferiore ad un'espressione della forma $\frac{m_{1} n_{n}^{n}}{|2|^{n}}, n<1$; perciò sarà anche :

$$
\left|k_{n+1}-c \frac{b_{0} b_{1} \ldots b_{n}}{a_{0} a_{1} \ldots a_{n+1}}\right|<\frac{m_{2} n^{n}}{|z|^{n}}
$$

e quindi :

$$
\left|\frac{b_{n}}{a_{n+1}} k_{n}-k_{n+1}\right|<\frac{\left(m_{1}+m_{2}\right) n^{n}}{|z|^{n}},
$$

che equivale alla condizione $\left(13^{\prime \prime}\right)$, cioè alla (10).

La condizione è sufficiente. Se infatti $\mathrm{i}$ coefficienti $k_{n}$ della serie $\varphi(x)=\Sigma k_{n} x^{n}$ soddisfano alla (13), ne verrà :

$$
\left|p_{n} k_{n}-z p_{n+1} k_{n+1}\right|<\frac{m\left|p_{n+1}\right| n^{n}}{|z|^{n-1}}
$$

e poichè la serie $\Sigma \frac{p_{3 z}}{z^{n}} x^{n}$ converge entro il cerchio $[z]$, la serie:

$$
\psi(x)=\Sigma\left(p_{n} k_{n}-z p_{n+1} k_{n+1}\right) x^{n}
$$


convergerà in un cerchio maggiore, cioè apparterrà ad $S_{z}$. E siccome la serie $\psi(x)$ non differisce sostanzialmente da $U_{1}^{-1} A(\varphi)$, la quale ha le singolarità negli stessi punti in cui le ha $A(\varphi)$, concludiamo che $A(\psi)$ appartiene ad $S_{z}$, c. d. d.

77. Il noto teorema del sig. Darboux $\left({ }^{*}\right)$, che dà la condizione necessaria e sufficiente affinchè una serie di potenze convergente entro [z] abbia sulla circonferenza, come sola singolarità, un polo semplice, si deduce immediatamente dal teorema del paragrafo precedente facendovi $p_{n}=1$. La condizione (13) si riduce a :

$$
\left|\sqrt[n]{\frac{k_{n}}{z}-k_{n+1}}\right|<a<\frac{1}{|z|}
$$

per $n>n_{1}$. Inoltre, da:

$$
\left|k_{n}-c \frac{b_{0} b_{1} \ldots b_{n-1}}{a_{0} a_{1} \ldots a_{n}}\right|<\frac{m_{1} \eta_{n}^{n}}{|z|^{n}}
$$

si deduce nel caso attuale:

onde :

$$
\left|k_{n} z^{n}-c\right|<m_{1} \eta^{n}, \quad n<1
$$

$$
\lim _{n=\infty} k_{n} z^{n}=c
$$

e queste sono appuntó le condizioni date dal Darboux.

78. L'operazione $A$ applicata ad una serie di potenze $\varphi=\Sigma k_{n} x^{n}$, riproduce dunque una serie di potenze avente in generale lo stesso cerchio di convergenza della serie $\varphi$, ed un cerchio maggiore solo quando $\varphi(x)$ converge in $[z]$ ed ha in $z$ la singolarità definita da $\omega(x)$. Applicata dunque ad una serie $\rho$ di $S_{z}$, la $A$ prodnce una serie $\rho_{1}$ pure di $S_{z}$. Ma i coefficienti di $\rho_{1}$ non sono totalmente arbitrarî; essi verificano una relazione necessaria. Si ha infatti (75):

con :

$$
A(\varphi)=\Sigma g_{n} x^{n}
$$

Ne viene :

$$
g_{n}=\Pi\left(k_{n}\right)=q_{n}\left(p_{n} z k_{n}-p_{n-1} k_{n-1}\right)
$$

$$
\frac{g_{n}}{q_{n}}=k_{n} p_{n} z-k_{n-1} p_{n-1}, \quad \frac{g_{0}}{q_{0}}=k_{0} p_{0},
$$

(*) Journal de Mathématiques, S. III, T. IV, 1878. V. a questo proposito il notevole riassunto del siy. Osgood, Bulletin of the Americ. Math. Sociely, S. II, Tom. V, pag. 59. 
da cui :

$$
\frac{g_{0}}{q_{0}}+\frac{g_{1}}{q_{1}} z+\cdots+\frac{g_{n}}{q_{n}} z^{n}=k_{n} p_{n} z^{n+1} .
$$

Ma $\Sigma k_{n} x^{n}$ è per ipotesi un elemento di $S_{z}$, e perciò $z^{n}$ appartiene all'interno del suo cerchio di convergenza; lo stesso accade anche per $\Sigma k_{n} p_{n} x^{n}$, e perciò :

$$
\lim _{n=\infty} k_{n} p_{n} z^{n+!}=0
$$

Ne viene che $\mathrm{i}$ coefficienti $g_{n}$ della serie $\rho_{4}=A(\varphi)$ soddisfano alla condizione:

$$
\underset{n=0}{\stackrel{\infty}{=}} g_{n} \frac{z^{n}}{q_{n}}=0, \quad \text { ossia } \quad g_{0}+\frac{a_{0}}{b_{0}} g_{1}+\frac{a_{0} a_{1}}{b_{0} b_{1}} g_{2}+\frac{a_{0} a_{1} a_{2}}{b_{0} b_{1} b_{2}} g_{3}+\cdots=0
$$

Si noti che la funzione $\frac{z^{n}}{q_{n}}$, che figura nel termine generale della serie $\rho_{1}$, è l'integrale dell'equazione $\bar{\Pi}\left(k_{n}\right)=0$, dove $\bar{\Pi}$ è la forma alle differenze $a g$ giunta $\left(^{*}\right)$ di $\Pi$.

79. Passiamo ora a cercare l'effetto prodotto dall' inversa $A^{-1}$ dell'operazione $A$ applicata ad un elemento di $S_{0}$. In questo insieme, come sappiamo, la $A^{-1}$ è a determinazione unica. Sia $\rho(x)$ una serie di $S_{z}$, e $\rho=\Sigma g_{n} x^{n}$.

Dalla (6) segue :

$$
A^{-1}=U^{-1} M_{\frac{1}{z-x}} U_{1}^{-1}
$$

e perciò :

$$
A^{-1}(\rho)=U^{-1}\left\{\frac{1}{z-x}\left(\frac{g_{0}}{q_{0}}+\frac{g_{1}}{q_{1}} x+\frac{g_{2}}{q_{2}} x^{2}+\cdots\right)\right\}
$$

La $U_{1}^{-1}(\rho)$ è pure una serie di $S_{z}$; invece $\frac{1}{z-x}$ ammettendo uno sviluppo in serie convergente entro $[z]$, lo stesso sarà della funzione soggetta all'operazione $U^{-1}$ nel secondo membro della (15), e lo stesso ancora sarà di $A^{-1}(\rho)$, il cui sviluppo è :

$$
A^{-1}(\rho)=\sum_{n=0}^{\infty}\left(\frac{g_{0}}{q_{0}}+\frac{g_{1} z}{q_{1}}+\frac{g_{2} z^{2}}{q^{2}}+\cdots+\frac{g_{n} z^{n}}{q_{n}}\right) \frac{x^{n}}{p_{n} z^{n+1}} .
$$

(*) V. la mia Nota: Sulloperazione aggiunta. Rendiconti dell'Accademia di Bologna, 17 aprile 1898 (\$ 11).

Annali di Matemalica, Serie III, tomo IV. 
Vi sarà eccezione nel solo caso che la serie

$$
U_{1}^{-1}(\rho)=\Sigma \frac{g_{n} x^{n}}{g_{n}}
$$

ammetta uno zero per $x=z$, cioè sotto la condizione:

$$
\frac{g_{0}}{q_{0}}+\frac{g_{1} z}{g_{1}}+\frac{g_{2} z^{2}}{g_{2}}+\cdots=0
$$

in questo solo caso $A^{-1}(\rho)$ apparterrà ad $S_{z}$.

80. Dal paragrafo precedente risulta che l'operazione $A^{-1}$, applicata ad una serie di $S_{z}$, dà una serie che, in generale, non converge oltre al cerchio $[z]$. L'operazione $A^{-1}$ introduce dunque una singolarità sulla circonferenza [z], e siccome questa singolarità è tolta da $A$, così essa è quella singolarità isolata nel punto $x=z$ il cui tipo è dato dalla serie:

$$
\omega(x)=\frac{1}{a_{0}}+\frac{b_{0} x}{a_{0} a_{1}}+\frac{b_{0} b_{1} x^{2}}{a_{0} a_{1} a_{2}}+\cdots
$$

Fra le operazioni $A^{-1}$, la più semplice è la divisione per $x-z$, che introduce nella serie di $S_{z}$, un polo di prim'ordine. In questo caso:

$$
A\left(x^{n}\right)=z x^{n}-x^{n+1}
$$

$a_{n}=z, b_{n}=1$, e la $\Sigma \frac{x^{n}}{z^{n+1}}$ dà il tipo della singolarità introdotta.

Risulta ancora dal paragrafo precedente che in quelle serie di $S_{z}$ i cui coefficienti soddisfano alla relazione (14), la $A^{-1}$ non introduce singolarità. Questa condizione (14), dimostrata sufficiente, è anche necessaria, poichè si è visto al $\S 78$ che se $\rho$ è una serie di $S_{z}, A(\rho)$ soddisfa, coi suoi coefficienti, alla relazione (14): onde inversamente se $A(\rho)$ non soddisfa a tale condizione, $\rho$ non può appartenere ad $S_{z}$.

La condizione (14) va dunque riguardata come una estensione della condizione di divisibilità di una serie di potenze per il binomio $x-z$, cui essa si riduce nel caso semplice dı $A=M_{x-z}$. Concludendo:

"L'operazione $A^{-1}$, applicata ad una serie di $S_{z}$, vi introduce in generale una singolarità rappresentata dalla $\omega(x)$. Condizione necessaria e sufficiente perchè questa singolarità non si introduca, ̀̀ che i coefficienti $g_{n}$ della serie soddisfino alla condizione (14)." 
atte ad aggiungere o togliere singolarità in una funzione analitica. 267

81. Per dare un'applicazione di quanto precede, consideriamo la forma differenziale lineare dell'ordine $m$ :

$$
F(\varphi)=\left(h_{0}-k_{0} x\right) \varphi+\left(h_{1}-k_{1} x\right) x \varphi^{\prime}+\cdots+\frac{1}{m !}\left(h_{m}-k_{m} x\right) x^{m} \varphi^{(m)} \text {. }
$$

L'equazione $F(\varphi)=0$ appartiene ad un tipo che è stato studiato dal sig. Goursat (*). Applicando la $F$ ad $x^{n}$, si ottiene :

$$
F\left(x^{n}\right)=\left(h_{0}+n h_{1}+\cdots\left(\begin{array}{c}
n \\
m
\end{array}\right) h_{m}\right) x^{n}-\left(k_{0}+n k_{1}+\cdots+\left(\begin{array}{c}
n \\
m
\end{array}\right) k_{m}\right) x^{n+1},
$$

ed $F$ è quindi un'operazione normale del primo ordine, del tipo (5), in cui :

$$
a_{n}=h_{0}+n h_{1}+\cdots\left(\begin{array}{c}
n \\
m
\end{array}\right) h_{m}, \quad b_{n}=k_{0}+n k_{1}+\cdots\left(\begin{array}{c}
n \\
m
\end{array}\right) k_{m} ;
$$

gli $a_{n}, b_{n}$ sono dunque polinomî razionali interi in $n$, del grado $m$, che noi supporremo diversi da zero pei valori interi positivi di $n$. Reciprocamente, ogni operazione del tipo (5) in cui $a_{n}, b_{n}$ sono razionali interi di grado $m$ in $n$ è una forma differenziale lineare del tipo (17).

Ciò posto, indicando con $z$ il rapporto $h_{m}: k_{m}$, risulta dai principî sull'integrazione delle equazioni differenziali lineari che l'equazione:

$$
F(\varphi)=\rho,
$$

dove $\rho$ è una serie di $S_{z}$, ammette una sola soluzione regolare nell' intorno di $x=0$ e precisamente entro [z], colla sola singolarita $x=z$ sulla circonferenza. L'operazione $F^{-1}$ introduce, l'operazione $F$ toglie cotesta singolarità.

Poniamo ora:

con :

$$
F=U_{\mathrm{i}} M U
$$

ne risulterà $(\$ 55)$ :

$$
U\left(x^{n}\right)=p_{n} x^{n}, \quad U_{1}\left(x^{n}\right)=q_{n} x^{n}
$$

$$
q_{n}=\frac{b_{0} b_{1} \ldots b_{n-1}}{a_{0} a_{1} \ldots a_{n-1}} z^{n}, \quad p_{n}=\frac{a_{0} a_{1} \ldots a_{n-1} a_{n}}{b_{0} b_{1} \ldots b_{n-1}} \frac{1}{z^{n+1}} .
$$

Dico che queste operazioni $U, U_{1}$ ammettono la proprietà di essere semplici, esse e le loro inverse. Considerando infatti la:

$$
U^{-1}\left(\frac{1}{z-x}\right)=\frac{1}{a_{0}}+\frac{b_{0} x}{a_{0} a_{1}}+\frac{b_{0} b_{1} x^{2}}{a_{0} a_{1} a_{2}}+\cdots=\omega(x),
$$

(*) Am. de l'École Normale, S. II, T. XII, 1883. 
ed applicandole l'operazione $F$, viene:

$$
F(\omega(x))=U_{1} M_{z-x} U U^{-1}\left(\frac{1}{z-x}\right)=1,
$$

quindi, per il ricordato teorema sulle equazioni differenziali lineari, $\omega(x)$ ha un'uniea singolarità a distanza finita, per $x=z$. Ne viene che $U^{-1}\left(\frac{1}{1-x}\right)$ a vrà un'unica singolarità per $x=1$ a distanza finita, ed $U^{-1}$ è quindi un'operazione semplice. Ma l'operazione $U$ dà ancora:

$$
U\left(\frac{1}{1-x}\right)=\Sigma \frac{a_{0} a_{1} \ldots a_{n}}{b_{0} b_{1} \ldots b_{n-1}} \frac{x^{n}}{z^{n+1}}=\varpi(x) .
$$

Applicandole l'operazione $F_{1}$ definita da :

$$
F_{1}(1)=z-a_{1} x, \quad F_{1}\left(x^{n}\right)=b_{n-1} z x^{n}-a_{n+1} x^{n+1}, \quad(n=1,2,3, \ldots),
$$

che è pure una forma differenziale lineare del tipo (17) col punto singolare $x=1$, troveremo $F_{1}(\varpi(x))=z a_{0}$; ne viene che $\sigma(x)$ ammette il solo punto singolare $x=1$ a distanza finita, e quindi anche $U$ è un'operazione semplice.

Analogamente risulta dalla forma dei coefficienti $q_{n}$ ed $\frac{1}{q_{n}}$ che $U_{1}$ e la sua inversa sono operazioni semplici, talchè la $F$ è un caso particolare dell'operazione $A$ studiata nei $\$ \S 67$ e seguenti.

82. Dal paragrafo precedente risulta che se $a_{n}, b_{n}$ sono polinomî razionali interi di grado $m$ in $n$, la serie:

$$
\omega(x)=\sum \frac{b_{0} b_{1} \ldots b_{n-1}}{a_{0} a_{1} \ldots a_{n}} x^{n}, \quad\left(\lim _{n=\infty} \frac{a_{n}}{b_{n}}=z\right),
$$

definisce una funzione analitica avente nel piano $x$ la sola singolarità $x=z$ a distanza finita. Se una serie $\varphi(x)=\Sigma k_{n} x^{n}$, avente [z] come cerchio di convergenza, sarà singolare come $\omega(x)$ sulla circonferenza [z], la $F$ corrispondente toglierà la singolarità alla $\varphi(x)$; in altri termini, dovranno i coeffcienti $k_{j}$ verificare la condizione necessaria e sufficiente:

$$
\left|a_{n} k_{n}-b_{n-1} k_{n-1}\right|<\frac{m n^{n}}{|z|^{n}}
$$

dove $m$ ed $n$ sono numeri positivi ed è $n<1$. L'operazione $F$ toglie quindi, e la $F^{-1}$ introduce, in generale, la singolarità rappresentata da $\omega(x)$; però, $F^{-1}$ non introduce questa singolarità in una serie di $S_{z}$, quando i coeffi- 
atte ad aggiungere o togliere singolarità in una funzione analitica. 269

cienti $g_{n}$ della serie soddisfano alla condizione (14):

$$
g_{0}+g_{1} \frac{a_{0}}{b_{0}}+g_{2} \frac{a_{0} a_{1}}{b_{0} b_{1}}+\cdots=0
$$

in cui il primo membro è una serie di funzioni razionali di $n$.

\section{Uso deLLE OPERAzIONI NoRMaLI D'ORDINE SUPERIORE NEL TOGLIERE SINGOLARITÀ.}

83. Consideriamo un'operazione normale $P$ di ordine $m$, definita nell'insieme $S$ ' dalle relazioni :

$$
P\left(x^{n}\right)=a_{n 0} x^{n}+a_{n 1} x^{n+1}+\cdots+a_{n \cdot m i} x^{n+m} .
$$

Abbiamo dimostrato al $\S 57$ che una simile operazione si può presentare sotto forma del produtto:

$$
P=U_{m} M_{z_{m}-x} U_{m-1} \ldots U_{1} M_{z_{1}-x} U_{0}
$$

dove $U_{0}, U_{\mathrm{s}}, \ldots U_{m}$ sono operazioni normali d'ordine nullo, definite in $S$ da:

$$
U_{i}\left(x^{n}\right)=p_{i n} x^{n},\left(\begin{array}{c}
i=0,1, \ldots m, \\
n=0,1,2, \ldots \infty
\end{array}\right) .
$$

Faremo ora le seguenti ipotesi :

a) che si siano trovati numeri opportuni $z_{1}, z_{2}, . . z_{m}$ pei quali le operazioni $U$ che figurano nella scomposizione (19) siano semplici insieme alle loro inverse;

b) che sia $\left|z_{1}\right|<\left|z_{2}\right|<\cdots<\left|z_{m}\right|$. La $z_{m}$, che ha il modulo massimo verrà indicata semplicemente con $z ; S_{z}$ rappresenterà ancora l'insieme delle serie di potenze di $z$ il cui raggio di convergenza è superiore a $|z|$; infine si userà la lettera $\rho$, affetta $o$ no da indici, per indicare le serie di $S_{z}$.

84. Sotto le ipotesi del paragrafo precedente, l'operazione $P$ applicata ad una serie di potenze $\varphi$ di cui $[r]$ è il cerchio di convergenza, dà una serie di potenze $\psi$ il cui cerchio di convergenza non può essere inferiore ad $[r]$, ma può, in alcuni casi, essere maggiore. Quando ciò accada, l'operazione $P$ avrà, per effetto " di togliere le singolarità della " $(x)$ sulla circonferenza $r . "$ Ci proponiamo di vedere quali singolarità possono effettivamente venire tolte dalla $P$. 
A quest'uopo, sia posto:

$$
A_{1}=M_{z_{1}-\infty} U_{0}, \quad A_{2}=M_{z_{2}-\infty} U_{1}, \ldots \quad A_{m}=U_{m} M_{z_{m}-x} U_{m-1},
$$

onde :

$$
P=A_{m} A_{m-1} \ldots A_{2} A_{1} \text {. }
$$

La formula (20) ci dà così una scomposizione di $P$ in un prodotto di $m$ operazioni normali del prim'ordine, della forma di quelle precedentemente studiate ( $\$ \$ 67$ e seg.). Sappiamo pertanto che ad ogni operazione $A_{i}$ corrisponde una serie di potenze $\omega_{i}$, la quale soddisfa all'equazione:

$$
A_{i}\left(\omega_{i}\right)=1
$$

posto :

la serie $\omega_{i}$ è data da:

$$
U_{i}\left(x^{n}\right)=p_{i, n} x^{n}
$$

$$
\omega_{i}(x)=\sum_{n=0}^{\infty} \frac{x^{n}}{p_{i-1 \cdot n} z_{i}^{n}} .
$$

e rappresenta una funzione analitica che a distanza finita, ha per solo punto singolare $x=z_{i}$; l'operazione $A_{i}^{-1}$ introduce e la $A_{i}$ fa sparire questa singolarità $(\$ 89)$. Consideriamo ora le funzioni :

$$
\left.\begin{array}{c}
\pi_{1}=\omega_{1}, \quad \pi_{2}=A_{1}^{-1}\left(\omega_{2}\right), \\
\pi_{3}=A_{1}^{-1} A_{2}^{-1}\left(\omega_{3}\right), \ldots \quad \pi_{m}=A_{1}^{-1} A_{2}^{-1} \ldots A_{m-1}^{-1}\left(\omega_{m}\right) .
\end{array}\right)
$$

Dico che la funzione $\pi_{i}$ ammette a distanza finita al più $i$ singolarità isolate nei punti $z_{1}, z_{2}, \ldots z_{i}$. Infatti, la $\pi_{1}$ non differisce da $\omega_{1}$ ed ammette la sola singolarità $z_{1}$ a distanza finita, La $\omega_{2}$ ammette la sola $z_{2}$ ed è quindi una serie di $S_{z_{1}}$; applicandovi la $A_{0}^{-1}=U_{0}^{-1} M_{\frac{1}{z_{1}-x}}$, non s'introduce altra singolarità che quella in $z_{1}$, e quindi $\pi_{2}$ ha (al più) sole singolarità isolate in $z_{1}$ e $z_{2}$ a distanza finita. Analogamente per $\pi_{3}, \ldots \pi_{i}$.

85. Applicando ora la operazione $P$ ad una serie di potenze della forma :

$$
\varphi(x)=c \pi_{i}(x)+\rho
$$

dove $\rho$, come è stabilito, è una serie di $S_{z}$, avremo per la (20):

$$
P(\varphi)=c A_{m} A_{m-1} \ldots A_{2} A_{1}\left(\pi_{i}\right)+P(\rho) ;
$$

ora $P(\rho)$ è una serie $\rho_{1}$, e, poichè :

$$
\pi_{i}=A_{1}^{-1} A_{2}^{-1} \ldots A_{i-1}^{-1}\left(\omega_{i}\right) \text { ed } A_{i}\left(\omega_{i}\right)=1
$$


atte ad aggiungere o togliere singolarità in una funzione analitica. 271

viene :

$$
P(\varphi)=c A_{m} A_{m-1} \ldots A_{i+1}(1)+\rho_{1} .
$$

In questo secondo membro, il primo termine è un polinomio razionale intero in $x$, al più del grado $m-i$; il secondo termine è una serie di $S_{z}$, onde $P(\varphi)$ è una serie di $S_{z}$ : cioè $P$ toglie le singolarità che $\pi_{i}$ ammette entro il cerchio $[z]$. Se ne conclude che "l'operazione $P$ toglie le singolarità rappresentate dalle funzioni $\pi_{1}, \pi_{2}, \ldots \pi_{m}$ : ciò̀, essendo $c_{1}, c_{2}, \ldots c_{m}$ costanti arbitrarie, la $P$, applicata a:

dà una serie $\rho . "$

$$
c_{1} \pi_{1}+c_{2} \pi_{2}+\cdots+c_{m} \pi_{m}+\rho
$$

Si avverta che $P\left(\pi_{m}\right)=1$, e che $P\left(\pi_{m-i}\right)$ è un polinomio di grado $i$ in $k$, a coefficienti facilmente calcolabili.

86. Reciprocamente a quanto è dimostrato nel paragrafo precedente, sia una serie di potenze cui $P$ tolga la singolarità in $[z]$; vale a dire la $\varphi$ non sia una serie $\varphi$, ma sia tale la $P(\varphi)$. Dico che $\varphi$ deve essere della forma (23). Se infatti $\dot{e} P(\varphi)=\rho$, ne viene:

$$
P^{{ }^{\prime}}(\rho)=A_{1}^{-1} A_{\%}^{-1} \ldots A_{m}^{-1}(\rho)=\varphi ;
$$

ora $A_{m i}^{-1}(\rho)$ non può avere singolarità in $[z]$ se non nel punto $z_{m}$, e sarà $(\S 80)$ :

da questa :

$$
A_{m}^{-1}(\rho)=c \omega_{m}+\rho_{1}
$$

$$
\begin{gathered}
A_{m-1}^{-1} A_{m}^{-1}(\rho)=c A_{m-1}^{-1}\left(\omega_{m}\right)+c_{1} \omega_{m-1}+\rho_{2}, \\
A_{m-2}^{-1} A_{m-1}^{-1} A_{m}^{-1}(\rho)=c A_{m-2}^{1} A_{m-1}^{-1}\left(\omega_{m}\right)+c_{1} A_{m-1}^{-1}\left(\omega_{m-1}\right)+c_{2} \omega_{m-2}+\rho_{3},
\end{gathered}
$$

e cos̀ via. Tenuto conto delle (22), si ottiene :

c. d. d.

$$
P^{-1}(\rho)=c \pi_{m}+c_{1} \pi_{m}+\cdots+c_{m-1} \pi_{1}+\rho_{m+1},
$$

87. Si avrà un esempio delle singolarità introdotte da $p^{-1}$ risolvendo l'equazione funzionale:

$$
P(\omega)=b_{0}+b_{1} x+b_{2} x^{2}+\cdots+b_{m-1} x^{m-1},
$$

rispetto alla funzione $\omega$. Posto $\omega=\Sigma k_{n} x^{n}$ e $P(\omega)=\Sigma g_{n} x^{n}$, sappiamo dal $\S 50$ che le relazioni fra le $g_{n}$ e le $k_{n}$ sono espresse da:

$$
g_{0}=a_{00} k_{0}, \quad g_{n}=\Phi\left(k_{n}\right)=a_{n 0} k_{n}+a_{n-1 \cdot 1} k_{n-1}+\cdots+a_{n-m \cdot m} k_{n m} .
$$


Per la determinazione dei coefficienti $k_{n}$, cioè per la risoluzione dell'equazione proposta, si tratterrà dunque semplicemente di risolvere l'equazione lineare alle differenze:

colle condizioni iniziali :

$$
\Phi\left(k_{n}\right)=0
$$

$$
\left.\begin{array}{c}
a_{00} k_{0}=b_{0}, \quad a_{10} k_{1}+a_{01} k_{0}=b_{1}, \ldots \\
a_{m-1 \cdot 0} k_{m-1}+a_{m-2 \cdot 1} k_{m-2}+\cdots+a_{0 \cdot m-1} k_{0}=b_{m-1} .
\end{array}\right)
$$

L'arbitrarietà dei coefficienti del secondo membro della (24) equivale a quella delle costanti nell' integrale della (26), e la determinazione delle une porta, linearmente, a quella degli altri.

88 Abbiamo così ottenuto il seguente risultato:

"Una serie $\Sigma k_{n} x^{n} \mathrm{j}$ cui coefficienti soddisfano all'equazione lineare alle differenze, d'ordine $m, \Phi\left(i_{n}\right)=0$, il cui primo membro è la trasformata $C P C^{-1}$ di $P$ mediante l'operazione $C$ definita al $\S 30$, rappresenta una funzione che ha entro il cerchio $[z]$ i soli punti singolari $z_{1}, z_{2}, \ldots z_{m}$, e le singolarità in questi punti sono tolte mediante l'applicazione della $P$."

Si ha ancora che per la serie $\Sigma_{n} x^{n}$ in discorso, il limite superiore dell'insieme derivato di $\left|\sqrt[n]{k_{n}}\right|$ è, in generale, $\frac{1}{\left|z_{1}\right|}$ e non è in nessun caso maggiore: potendosi ridurre, per una scelta opportuna delle $b_{0}, b_{1}, \ldots b_{m-1}$, ad essere $\frac{1}{\left|z_{i}\right|}, i>1\left(^{*}\right)$.

89. Dalla scomposizione di $P$ in fattori nella forma (20) segue che anche la sua trasformata $C P C^{-1}=\Phi$ si scompone nella forma:

$$
\Phi=\Pi_{m} \Pi_{m-1} \ldots \Pi_{z} \Pi_{1}
$$

dove $\Pi_{i}$ è la forma lineare alle differenze del prim'ordine,

$$
\Pi_{i}=z_{i} p_{i-1, n} k_{n}-p_{i-i, n-1} k_{n-1}, \quad(i=1,2, \ldots m-1),
$$

mentre è :

$$
\Pi_{m}=p_{m . n}\left(z_{m} p_{m-1, n} k_{n}-p_{m-1, n-1} k_{n-1}\right) .
$$

I coefficienti della soluzione $\omega$ dell' equazione (24) si ottengono dall'integrazione della equazione $\Phi=0$; ora questa integrazione è ricondotta a quella delle forme $n_{i}$ di prim'ordine, poichè indicando con

$$
h_{n}^{\prime}, \quad h^{\prime \prime}, \ldots h_{n}^{(m)},
$$

(*) Ofr. PoIncaré, Americ. Journal of Math., T. VII, 1885 (\$ 2). 
atte ad aggiungere o togliere singolarità in una funzione analitica. 273

gl'integrali di $\Pi_{1}=0, \Pi_{2}=0, \ldots \Pi_{m}=0$ rispettivamente, si vede immediatamente che un sistema fondamentale di integrali di $\Phi=0$ sarà dato da:

$$
k_{n}^{\prime}=h_{n}^{\prime}, \quad k_{n}^{\prime \prime}=\Pi_{1}^{-1}\left(h^{\prime \prime}{ }_{n}\right), \quad k^{\prime \prime \prime}{ }_{n}=\Pi_{1}^{-1} \Pi_{2}^{-1}\left(h^{\prime \prime \prime}{ }_{n}\right), \ldots
$$

90. Se si applica l'operazione $P$ ad una serie $\rho$, si ritrova una serie $\rho_{1}$. Ma quest'ultima ha coefficienti che soddisfano ad un certo numero di relazioni necessarie. Sia infatti $k_{n}$ il coefficiente di $x^{n}$ in $\rho$, e $g_{n}$ quello di $x^{n}$ in $\rho_{1}$; si avrà :

Si ponga:

$$
g_{n}=\Pi_{m} \Pi_{m-1} \ldots \Pi_{2} \Pi_{1}\left(k_{n}\right) \text {. }
$$

$$
\Pi_{m-1} \Pi_{m-2} \ldots \Pi_{1}\left(k_{n}\right)=g_{n}^{\prime}
$$

anche $\Sigma g_{n}^{\prime} x^{n}$ sarà una serie $\rho$, e si avrà :

$$
g_{n}=\Pi_{m}\left(g_{n}^{\prime}\right)
$$

onde, in forza del $\S 78$, se $t_{n}^{(m)}$ è l'integrale dell'equazione alle differenze $\bar{\Pi}_{m}=0$, aggiunta di $\Pi_{m}$, si avrà :

Si ponga poi :

$$
\Sigma g_{n} t_{n}^{(m)}=0 \text {. }
$$

$$
\Pi_{m-2} \Pi_{m-3} \ldots \Pi_{1}\left(k_{n}\right)=g_{n}^{\prime \prime},
$$

e si indichi con $\bar{\Pi}_{i}$ l'aggiunta di $\Pi_{i}$; considerando l'equazione $\bar{\Pi}_{m-1} \bar{\Pi}_{m}=0\left({ }^{*}\right)$, aggiunta di $\Pi_{m} \Pi_{m-1}$, un sistema fondamentale dei suoi integrali risulti costituito da $t_{z}^{(m)}$, integrale di $\overline{\boldsymbol{\Pi}}_{m}=0$, e da un altro $t_{n}^{\prime n-1)}$ : per quest'ultimo sarà :

$$
\bar{\Pi}_{n-1} \bar{\Pi}_{m}\left(t_{n}^{(m-1)}\right)=0,
$$

e siccome $t_{n}^{(m-1)}$ non annulla $\bar{\Pi}_{m}, \bar{\Pi}_{m}\left(t_{n}^{(m-1)}\right)$ è integrale di $\bar{\Pi}_{m-1}=0$. Ora si ha:

$$
g_{n}^{\prime}=\Pi_{m-1}\left(g^{\prime \prime}{ }_{n}\right)
$$

onde per il citato $\S 78$, sarà :

$$
\Sigma g_{n}^{\prime} \bar{\Pi}_{m}\left(t_{n}^{(m-1)}\right)=0 .
$$

Ma per le proprietà dell'operazione aggiunta $\bar{K}$ di una forma lineare alle differenze $K$, si ha $\left({ }^{* *}\right)$ :

$$
\Sigma K\left(a_{n}\right) b_{n}=\Sigma a_{n} \bar{K}\left(b_{n}\right),
$$

(*) Nota citata: Sulloperazione aggiunta, $\$ 6$.

(*) Ibid., $\$ 3$. 
onde la (30) potrà scriversi :

ossia :

$$
\Sigma \Pi_{m}\left(g^{\prime}\right) t_{n}^{(m-1)}=0,
$$

$$
\Sigma g_{n} t_{n}^{(m-1)}=0 \text {. }
$$

Così continuando, si vede che le $g_{n}$ soddisfano alla relazione:

$$
\Sigma g_{n} t_{n}=0
$$

essendo $t_{n}$ un integrale qualunque dell'equazione:

$$
\bar{\Phi}=\bar{\Pi}_{1} \bar{\Pi}_{2} \ldots \bar{\Pi}_{m}=0
$$

aggiunta di $\Phi=0$. Siccome tale integrale contiene $m$ costanti arbitrarie, così la (31) equivale ad $m$ relazioni indipendenti della stessa forma.

91. Rimane ora da vedere che le serie $\Sigma g_{n} t_{n}$ che figurano nel paragrafo precedente sono assolutamente convergenti, essendo $t_{n}$ un integrale qualunque dell'equazione (32). Ciò si dimostrerà provando che è assolutamente convergente la serie $\Sigma g_{n} u_{n}$, dove $g_{n}$ sono i coefficienti di una serie $\rho$ ed $u_{n}$ l'integrale di una qualunque delle equazioni $\bar{\Pi}_{i}=0$. Ora se si ha in generale:

ne viene $(*)$ :

$$
\Pi\left(k_{n}\right)=\left(p_{n+1} k_{n+1} z_{i}-p_{n} k_{n}\right) q_{n}
$$

$$
\bar{\Pi}\left(k_{i n}\right)=p_{n}\left(q_{n-1} k_{n-1} z-q_{n} k_{n}\right)
$$

L'integrale di quest'ultima forma è $u_{n}=\frac{z_{i}^{n}}{q_{n}}$ : ora, per le proprietà di $q_{n}$, poichè $\Sigma g_{n} x^{n}$ converge in un cerchio maggiore di $\left[z_{m}\right]$, e $\left|z_{i}\right| \leq\left|z_{m}\right|$, segue che $\Sigma g_{n} u_{n}$ è assolutamente convergente.

92. Dalle cose dette, si conclude:

a) che l'applicazione dell'operazione $P$ ad una serie $\rho$ di $S_{z}$ produce una serie $\rho_{1}$ di $S_{z}$; non però qualunque, poichè i coefficienti della $\rho_{1}$ soddisfảno ad $m$ relazioni indipendenti della forma (31).

b). Che l'applicazione di $P$ ad una serie $\varphi$ non appartenente ad $S_{z}$ produce una serie di $S_{z}$ se, e soltanto se la funzione $\varphi$ ammette entro $[z]$ $\mathrm{i}$ soli punti $z_{1}, z_{2}, \ldots z_{m}$ come punti singolari, con singolarità della forma (23).

(*) Sull'operazione aggizunla, $\$ 11$. 
alte ad aggiungere o togliere singolarità in una funzione analitica. 275

\section{1)i conseguenza:}

c) L'applicazione dell'operazione $P^{-1}$ ad una serie $\rho$ di $S_{z}$ produrrà in generale una serie non appartenente ad $S_{z}$; affinchè $\varphi$ appartenga ad $S_{z}$ devono necessariamente essere soddisfatte le condizioni (31) dai coefficienti di $\rho$.

d) Queste condizioni sono anche sufficienti affinchè $P^{-1}(\rho)$ sia una serie $\rho_{1}$. Infatti, sia $\rho=\Sigma g_{n} x^{n}$ una serie $i$ cui coefficienti soddisfino alla condizione (31) per ogni integrale $t_{n}$ dell'equazione $\bar{\Phi}=0$. Applicando dapprima a $\rho$ l'operazione $A_{m}^{-1}$, si abbia :

$$
A_{m}^{-1}(p)=\Sigma g_{n}^{\prime} x^{n}=\lambda_{1} \text {. }
$$

Ne viene $A_{m}\left(\lambda_{1}\right)=\rho$, ossia $\Pi_{m}\left(g_{n}^{\prime}\right)=g_{n}$; ma poichè le $g_{n}$ soddisfano alla (31) per ogni integrale di $\bar{\Phi}=0$, sarà anche $\Sigma g_{n} t_{n}^{(1)}==0$ per quell'integrale $t_{n}^{(1)}$ che annulla $\Pi_{m}$. Perciò, per il teorema del $\S 80, A_{m}^{-1}$ applicata a $\rho$ non introduce singolarità, cioè $\lambda_{1}$ è una serie di $S_{z}$.

Considero ora $A_{m-1}^{-1}\left(\lambda_{1}\right)=\Sigma g_{n}^{\prime \prime} x^{n}=\lambda_{2}$ : dico che anche $\lambda_{2}$ è una serie di $S_{z}$. Infatti, per l'jpotesi fatta sulle $g_{n}$, sarà anche $\Sigma g_{n} t_{n}^{(2)}=0$ per quell'integrale $t_{n}^{(2)}$ della (32) per il quale è nullo $\bar{\Pi}_{m-1} \bar{\Pi}_{m}$. Si ha cioè che

$$
\bar{\Pi}_{m}\left(t_{22}^{(2)}\right)=s_{n}
$$

è integrale di $\bar{\Pi}_{m-1}=0$. Ma si può scrivere, per le proprietà delle operazioni aggiunte :

$$
0=\Sigma g_{n} t_{n}^{(2)}=\Sigma \Pi_{m}\left(g_{n}^{\prime}\right) t_{n}^{(2)}=\Sigma g_{n}^{\prime} \bar{\Pi}_{m}\left(t_{n}^{(2)}\right),
$$

ossia :

$$
\Sigma g_{n}^{\prime} s_{n}=0
$$

onde è soddisfatta la condizione del teorema del $\S 80$ perchè $A_{m-1}^{-1} \lambda_{1}=\lambda_{2}$ sia una serie di $S_{z}$. Così continuando, si giunge alla conclusione che:

$$
A_{m-2}^{-1} A_{m-1}^{-1} A_{m}^{-1}(\rho), \ldots A_{2}^{-1} A_{3}^{-1} \ldots A_{m}^{-1}(\rho),
$$

ed infine $P^{-1}(\rho)=A_{1}^{-1} A_{2}^{-1} \ldots A_{m}^{-1}(\rho)$, sono serie di $S_{z}$, c. d. d.

93. L'operazione $P$ definita dalle (18) ha dunque la proprietà di togliere da una funzione analitica un certo complesso di singolarità; la $P^{-1}$ introduce invece queste singolarità quando essa si applichi ad una serie di $S_{z}$. Le condizioni (31), cui soddisfano i coefficienti di quelle serie di $S_{z}$ nelle quali, per eccezione, la $P^{-1}$ non introduce singolarità, sono analoghe alle condizioni di divisibilità di una serie per un polinomio, e si riducono a quelle 
condizioni quand, tutte le operazioni $U$ che figurano nella (19) si riducono all'operazione identica.

94. Siano ancora $\pi_{1}, \pi_{2}, \ldots \pi_{m}$ le funzioni che definiscono le singolarità che $P$ è suscettibile di togliere. Se si pone l'equazione funzionale, dove $\rho$ è una serie data:

si avrà, per il $\S 86$ :

$$
P(\varphi)=\rho, \quad 0 \quad \varphi=P^{-1}(\rho)
$$

$$
\varphi=c_{1} \pi_{1}+c_{2} \pi_{2}+\cdots+c_{m} \pi_{m}+\rho_{1} .
$$

L'applicazione di $P$ ai due membri di questa eguaglianza darà un polinomio razionale intero di grado $m-1$, più la funzione $P\left(p_{1}\right)=p_{2}$. Talchè :

$$
P(\varphi)=\rho_{2}+d_{0}+d_{1} x+d_{2} x^{2}+\cdots+d_{m-1} x^{m-1}=\rho .
$$

La serie $\rho_{2}$, essendo il risultato di $P\left(\rho_{1}\right)$, sarà una serie i cui coefficienti soddisfano alle condizioni (31); tale è dunque anche la :

$$
\rho-\left(d_{0}+d_{1} x+\cdots+d_{m-1} x^{m-1}\right)
$$

Se dunque la serie data $\rho$ è, ad esempio, la $\Sigma h_{n} x^{n}$, si avrà :

$$
\begin{gathered}
\rho-\left(d_{0}+d_{1} x \cdots+d_{m-1} x^{m-1}\right)= \\
=h_{0}-d_{0}+\left(h_{1}-d_{1}\right) x+\cdots\left(h_{m-1}-d_{m-1}\right) x^{m-1}+\sum_{n=m}^{\infty} h_{n} x^{n},
\end{gathered}
$$

e dovrà essere, indicando con:

$$
t_{n}^{\prime}, t^{\prime \prime}, \ldots t_{n}^{(m)}
$$

un sistema fondamentale d'integrali della $\bar{\Phi}=0$ :

$$
\begin{gathered}
\left(h_{0}-d_{0}\right) t_{0}^{(i)}+\left(h_{1}-d_{1}\right) t_{1}^{(i)}+\cdots+\left(h_{m-1}-d_{m-1}\right) t_{m-1}^{(i)}+h_{m} t_{m}^{(i)}+\cdots=0 \\
(i=1,2, \ldots m) .
\end{gathered}
$$

Queste equazioni servono a determinare $\mathrm{i}$ coefficienti $d_{0}, d_{1}, \ldots d_{m-1}$, e mediante questi $(\S 85)$ le costanti $c_{1}, c_{2}, \ldots c_{m}$, ciò̀ la parte singolare entro $[z]$ della $P^{-1}(\rho)$.

\section{Applicazione alLe equazioni differenziatil lineari.}

95. Come applicazione di quantn precede, consideriamo l'operazione rappresentata dal primo membro di un'equazione differenziale lineare del tipo di Fuchs, e che dicesi forma differenziale lineare normale. Per ottenere una 
atte ad aggiungere o togliere singolarità in una funzione analitica. 277

semplificazione di serittura che non altera la sostanza della questione, ci limiteremo al caso di una forma differenziale lineare, d'ordine qualunque $q$, a coefficienti trinomî, e sia:

$$
F(\varphi)=\sum_{i=0}^{q}\left(h_{i}+h_{i}^{\prime} x+h^{\prime \prime}{ }_{i} x^{2}\right) \frac{x^{i}}{i !} \varphi^{(i)}
$$

Formando $F\left(x^{n}\right)$, si ha:

dove :

$$
F\left(x^{n}\right)=\left(a_{n}+a_{n}^{\prime} x+a_{n}^{\prime \prime} x^{2}\right) x^{n}
$$

$$
a_{n}=h_{0}+h_{1} n+h_{2}\left(\begin{array}{l}
n \\
2
\end{array}\right)+\cdots+h_{q}\left(\begin{array}{l}
n \\
q
\end{array}\right)
$$

ed analoghe espressioni si hanno per $a_{n}^{\prime}$ ed $a_{n}^{\prime \prime}$. Si supporranno diversi da zero $a_{n}$ ed $a_{n}^{\prime \prime}$ per $n=0,1,2, \ldots$; si supporranno ancora di modulo diverso le radici $z$ e $z^{\prime}$ dell'equazione:

e precisamente:

$$
h_{q}+h_{q}^{\prime} x+h_{q}^{\prime \prime} x^{2}=0
$$

$$
\left|z^{\prime}\right|<|z| \text {. }
$$

I punti singolari dell'equazione differenziale lineare omogenea:

$$
F(\varphi)=0 \text {, }
$$

saranno $x=0, z^{\prime}, z$. L'equazione differenziale lineare non omogenea:

$$
F(\varphi)=c+c^{\prime} x
$$

avrà nell'intorno del punto $x=0$ un integrale (ed uno solo) sviluppabile in serie di potenze intere positive di $x$; sia $\omega\left(x ; c, c^{\prime}\right)$ questo sviluppo, che convergerà in generale entro il cerchio $\left[z^{\prime}\right]$, ed eccezionalmente entro il cerchio $[z]$.

96. Dico ora che è possibile di determinare le costanti $c, c^{\prime}$ per modo che $\omega\left(x ; c, c^{\prime}\right)$ ci rappresenti una funzione analitica semplice $(\$ 66)$ col solo punto singolare $z^{\prime}$ a distanza finita. Si ba intanto:

$$
\omega\left(x ; c, c^{\prime}\right)=c \omega(x ; 1,0)+c^{\prime} \omega(x ; 0,1) .
$$

Ora, nel piano della variabile $x$ conduciamo un taglio lungo una linea $l$ che vada da $z^{\prime}$ all'infinito senza passare per $z$ nè penetrare entro il cerchio $\left[z^{\prime}\right]$; nel piano cosi tagliato, $\omega(x ; 1,0)$ ed $\omega(x ; 0,1)$ sono funzioni analitiche singolari nel punto $z$, in cui la loro singolarità è rappresentata da:

$$
(x-z)^{e} \eta(x)
$$


essendo $\varepsilon$ la radice non nulla dell'equazione determinante della (35) rispetto al punto $z$, e l'espressione (37) essendo l'integrale canonico relativo. Si avranno dunque nell'intorno del punto $z$, i seguenti sviluppi per le funzioni $\omega(x ; 1,0)$ ed $\omega(x ; 0,1)$ :

$$
\begin{aligned}
& a(x ; 1,0)=e(x-z)^{\varepsilon} n(x)+\Re(x-z), \\
& \omega(x ; 0,1)=e_{1}(x-z)^{\varepsilon} n(x)+P_{1}(x-z),
\end{aligned}
$$

essendo $e$ ed $e_{1}$ due costanti e $\mathcal{H}, F_{1}$ sviluppi in serie di potenze. Prese dunque $\bar{c}$ e $\overline{c^{\prime}}$ per modo che sia :

viene :

$$
\bar{c} e+\bar{c} e_{1}=0
$$

$$
\omega\left(x ; \bar{c}, \overline{c^{\prime}}\right)=\bar{c} B(x-z)+\overline{c^{\prime}} B_{1}(x-z)
$$

abbiamo ciò̀ un'equazione (36) che ammette un integrale il quale non ha più singolarità per $x=z$.

Rappresenteremo quest' integrale $\omega(x ; \bar{c}, \bar{c})$ semplicemente con $\omega(x)$; la $\omega(x)$ è un ramo di funzione analitica che nel piano tagliato nel modo indicato non ha singolarità fuori di $l$, ed è quindi una funzione semplice. Come tale, essa caratterizza in gencrale ( $\$ 73$ ) una operazione normale di primo ordine $A_{1}=M_{z^{\prime}-\infty} U_{0}$, il cui effetto è di togliere la singolarità definita da $\omega(x)$ nel punto $z^{\prime}$.

Ottenuta la $A_{1}$, riprendiamo la forma $F$ e col metodo indicato al $\S 58$ determiniamo l'operazione normale di prim'ordine $A_{2}$ tale che sia:

$$
F=A_{2} A_{1} \text {. }
$$

Intanto $A_{1}$ toglie la singolarità rappresentata da $\omega(x)$. Ma ogni altro integrale della (36) ha quella stessa singolarità in $z^{\prime}$ : ne viene che se $\omega_{1} \grave{e}$ un altro integrale della (36) per valori arbitrarî delle $c, c^{\prime}$, la $A_{1}\left(\omega_{1}\right)=\pi$ non sarà singolare se non nel punto $z$. Sarà dunque $\pi$ una funzione semplice: ma

$$
F\left(\omega_{1}\right)=A_{2}(\pi)=c+c^{\prime} x
$$

non avendo singolarità a distanza finita, la singolarità di $\pi$ in $z$ è tolta da $A_{2}$. La forma differenziale lineare $F$ è dunque scomposta, nel modo indicato dalla formula (20), in un prodotto di due fattori di cui il primo $A_{1}$ toglie la singolarità $\omega$, il secondo la singolarità $\pi$; tale forma $F^{\prime}$ appartiene cioè alla specie delle operazioni $P$ studiate nei $\S \S 84$ e seguenti. 
atte ad aggiungere o togliere singolarità in una funzione analitica. 279

97. L'operazione $C$ definita al $\S 30$ trasforma l'operazione $F$ nella $C F^{\prime} C^{-1}=\Phi$ data da :

$$
\Phi\left(k_{n}\right)=a_{n} k_{n}+a_{n-1}^{\prime} k_{n-1}+a_{n-2}^{\prime \prime} k_{n-2},
$$

forma lineare alle differenze di second'ordine, i cui coefficienti sono polinomî in $n$ del grado $q$. La sua forma aggiunta ̀̀ :

$$
\bar{\Phi}\left(k_{n}\right)=a_{n} k_{n}+a_{n}^{\prime} k_{n+1}+a_{n}^{\prime \prime} k_{n+2} \text {. }
$$

Alla scomposizione di $F$ in fattori $\dot{A}_{1}, A_{2}$, corrisponde la scomposizione di $\Phi$ e di $\bar{\Phi}$ in prodotto di due forme lineare alle differenze del prim'ordine. Sia $r_{n}, r_{n}^{\prime}$ un sistema fondamentale d'integrali di $\bar{\Phi}=0$ : l'operazione $F$ applicata ad una serie $\rho$ di $S_{z}$, darà una serie di $\rho_{1}$ pure di $S_{z}$ i cui coefficienti $g_{n}$ soddisfaranno alle condizioni :

$$
\sum_{n=0}^{\infty} g_{n} r_{n}=0, \quad \sum_{n=0}^{\infty} g_{n} r^{\prime}{ }_{n}=0 \text {. }
$$

Queste sono anche le condizioni sufficienti affinchè l'equazione differenziale lineare non omogenea $F(\varphi)=\rho_{1}$ ammetta come soluzione una serie $\rho$ di $S_{z}$,

98. Risulta da quanto precede che ogni funzione singolare come un integrale dell'equazione (36), è tale che le sue singolarità sono tolte mediante l'applicazione dell'operazione $F$. Dico ora che, reciprocamente, se $\phi$ è una funzione cui la forma $F$ tolga le singolarità che essa ammette entro [z], queste singolarità sono quelle stesse dell'integrale $\omega\left(x ; c, c^{\prime}\right)$ della $(36)$. Infatti, essendo o una serie di $S_{z}$, si abbia:

$$
F(\varphi)=\rho=\Sigma g_{n} x^{n}
$$

$O$ i coefficienti $g_{n}$ soddisfano alle condizioni (38), e allora $\varphi$ è una serie $\rho_{1}$ di $S_{z}$, cioè non ha singolarità entro [z]. 0 esse non sono verificate; allora si possono determinare le costanti $d$ e $d^{\prime}$ (v. $\S 94$ ) in modo che sia:

$$
\begin{aligned}
& d r_{0}+d^{\prime} r_{1}=\sum_{0}^{\infty} g_{n} r_{n}, \\
& d r_{0}^{\prime}+d^{\prime} r_{1}^{\prime}=\sum_{0}^{\infty} g_{n} r_{n}^{\prime},
\end{aligned}
$$

e alle (38) soddisfaranno i coefficienti della serie $\rho-d-d^{\prime} x$. L'equazione:

$$
F(\varphi)=\rho-d-d^{\prime} x
$$


280 Pincherle: Di alcune operazioni atte ad aggiungere, ecc.

avrà dunque come integrale una serie $p_{1}$; ma l'equazione:

$$
F^{\prime}(\varphi)=d+d^{\prime} x
$$

ha per integrale la $\omega(x ; d, d)$; onde l'integrale della equazione proposta $F(\varphi)=\rho$ sarà la funzione:

$$
\varphi=p_{1}+\infty\left(x ; d, d^{\prime}\right)
$$

la quale ammette entro il cerchio [z] la singolarità caratterizzata da $\omega(x ; d, d)$. 